\title{
Exact Outage Probability of Two-Way Decode-and-Forward NOMA Scheme with Opportunistic Relay Selection
}

\author{
Tan-Phuoc Huynh ${ }^{1,2}$, Pham Ngoc Son ${ }^{3 *}$ and Miroslav Voznak ${ }^{1}$ \\ ${ }^{1}$ VSB Technical University of Ostrava, Czech Republic \\ [e-mail: phuoc.huynh.tan.st@vsb.cz,miroslav.voznak@vsb.cz] \\ ${ }^{2}$ Eastern International University (EIU), Binh Duong, Vietnam \\ [e-mail: phuoc.huynh@eiu.edu.vn] \\ ${ }^{3}$ Ho Chi Minh City University of Technology and Education, Ho Chi Minh city, VietNam \\ [e-mail: sonpndtvt@hcmute.edu.vn] \\ *Corresponding author: Pham Ngoc Son
}

Received December 27, 2017; revised January 7, 2019; accepted July 18, 2019;

published December 31, 2019

\begin{abstract}
In this paper, we propose a two-way relaying scheme using non-orthogonal multiple access (NOMA) technology. In this scheme, two sources transmit packets with each other under the assistance of the decode-and-forward (DF) relays, called as a TWDFNOMA protocol. The cooperative relays exploit successive interference cancellation (SIC) technique to decode sequentially the data packets from received summation signals, and then use the digital network coding (DNC) technique to encrypt received data from two sources. A max-min criterion of end-to-end signal-to-interference-plus-noise ratios (SINRs) is used to select a best relay in the proposed TWDFNOMA protocol. Outage probabilities are analyzed to achieve exact closed-form expressions and then, the system performance of the proposed TWDFNOMA protocol is evaluated by these probabilities. Simulation and analysis results discover that the system performance of the proposed TWDFNOMA protocol is improved when compared with a conventional three-timeslot two-way relaying scheme using DNC (denoted as a TWDNC protocol), a four-timeslot two-way relaying scheme without using DNC (denoted as a TWNDNC protocol) and a two-timeslot two-way relaying scheme with amplify-and-forward operations (denoted as a TWANC protocol). Particularly, the proposed TWDFNOMA protocol achieves best performances at two optimal locations of the best relay whereas the midpoint one is the optimal location of the TWDNC and TWNDNC protocols. Finally, the probability analyses are justified by executing Monte Carlo simulations.
\end{abstract}

Keywords: non-orthogonal multiple access (NOMA), digital network coding, cooperative communication, two-way transmission, decode-and-forward, outage probability. 


\section{Introduction}

In recent years, wireless networks have become one of the most common communication methods due to its flexibility in different environments. With the development of smart devices, internet services and advanced multimedia applications such as mobile TV and online game have surged to increase amounts of wireless connections [1-5]. To advance the data rate and the consumed energy in the next generation of wireless communications, non-orthogonal multiple access (NOMA) has been recently received great considerations from the researches in wireless systems as a promising technology to improve spectrum efficiency. The power domain NOMA is one of the popular operation methods where multiple-access users are allocated with different transmit powers although the same time and frequency [1]. Transmit signals of source users are combined by superposition coding, and destination users apply successive interference cancellation (SIC) to subtract co-channel interferences and decode desired data [6]. The authors in $[1,6]$ showed that the NOMA technology helps to improve the system throughput and to decrease transmission latency in wireless communications.

In order to deploy NOMA in a range of wireless systems, it is needed to combine with cooperative communications. In recent years, there are a lot of researches about cooperative communications to improve diversity capacity, and hence to increase the coverage and rate of wireless networks [7-10]. In the first timeslot, the sources broadcast their data to the relays while in the second timeslot, the relays assist the sources to transfer the received signals to the destinations by solutions as amplify-and-forward (AF) and decode-and-forward (DF) relaying techniques [11-14]. The opeartion of the DF technique is to decode data from received signals and forward re-coded data to the intended destinations whereas the relays in the AF technique only amplify the received data-carried signals and forward all to the destinations. As a result, the AF technique avoids the difficulty of the decoding operations but experiences from the noise addition caused by the amplification of both desired data-carried signals and noise. A combination of cooperative communications and NOMA is researched in [15-23]. J. B. Kim and I. H. Lee studied achievable average rate analyses of NOMA-applied relaying schemes [15]. S. Lee et al. in [18] investigated the system performance of NOMA-based AF relaying schemes in which the partial relay selection is used to obtain the best cooperative relay.

Two-way relaying protocols in [24-26] are investigated to improve the spectral utilization efficiency and enlarge the radio coverage of the wireless networks in which the sources interchange data via middle relays. The physical network coding such as digital network coding (DNC) and analog network coding (ANC) is a modern signal combination to decrease the number of transmission timeslots in the two-way cooperation protocols. Therefore, the physical network coding enhances bandwidth exploitation efficiency. In the DNC, the cooperative relays mix received data from the sources in the first and second timeslot by XOR procedure before forwarding coded data back to these sources in the third timeslot [24-25]. Whereas the relays in the ANC only use two timeslots and simply amplify received data-carried signals of the sources in the first timeslot, then these sources decode the desired data from the amplified version at the remaining timeslot [26]. The authors in [27-32] researched the two-way DF relaying networks and analyzed the system performances in terms of bit error rate (BER) [27], symbol error rate (SER) [28], block-error-rate (BLER) [29], maximum achievable sum-rate [30], frame error rate [31] and (sum) outage probability [32]. Opportunistic relay selections have been considered in [31-32] with different operating conditions to achieve maximum end-to-end signal quality. P. N. Son and H. Y. Kong in [33] 
investigated the performance improvements of two-way DF schemes by a combination of energy harvesting and DNC relays. A few researches for considering the NOMA technology two-way cooperative communications have been discussed to increase spectrum utilization efficiency [34-35]. However, the authors in these researches only use a relaying node to support packet transmission between two sources.

Encouraged by the above discussed problems, in this paper, we propose a two-way cooperative NOMA scheme with multi DF relays to enhance the spectral utilization efficiency where the best relay owning a maximum end-to-end signal-to-interference-noise ratio (SINR) is selected (called as a TWDFNOMA protocol) to assist two sources and using the SIC and DNC technology solutions to decode and encode received data from these sources.

The highlight contributions of our paper are given as the following results. Firstly, we propose the TWDFNOMA protocol where the best relay is found by the opportunistic relay selection method considering end-to-end SINRs. Secondly, exact closed-form expressions of (sum) outage probabilities are solved and then are validated by Monte Carlo simulations. Thirdly, the proposed TWDFNOMA protocol is better than a conventional three-timeslot two-way relaying scheme using DNC (called as a TWDNC protocol), a four-timeslot two-way relaying scheme without using DNC (called as a TWNDNC protocol) and a two-timeslot two-way relaying scheme with AF operations (called as a TWANC protocol). In addition, the system performance of the proposed TWDFNOMA protocol is improved when we have more cooperative relays.

The organization of this paper is showed as follows. Section 2 describes a multi-relay two-way system model and operation principle of the proposed TWDFNOMA protocol; The exact closed-form outage probability expressions of the proposed TWDFNOMA protocol are performed in Section 3; the simulation results of the proposed TWDFNOMA protocol and existing comparison protocols TWDNC, TWNDNC and TWANC are presented in Section 4; and our conclusions are summarized in Section 5.

\section{System model}

Fig. 1 presents a system model of a two-way relaying NOMA scheme with multi-wireless DF relays denoted as $R_{i}(i=1,2, \ldots, M)$, called as the TWDFNOMA protocol. In this figure, two sources $S_{1}$ and $S_{2}$ transmit their packets $x_{1}$ and $x_{2}$, respectively, to each other through the intermediate relays $R_{i}$. To achieve optimal packet transmission, a best relay $R_{b}$ using the NOMA technology is selected to exchange packets between two sources. We have some initial assumptions as 1) sources $S_{1}, S_{2}$ and relays $R_{i}$ are configured with a single antenna; 2) variances of zero-mean Additive White Gaussian Noises (AWGN) are identical, denoted as $N_{0}$; and 3) all channels are suffered to flat and block Rayleigh fadings and do not change during one transmission timeslot.

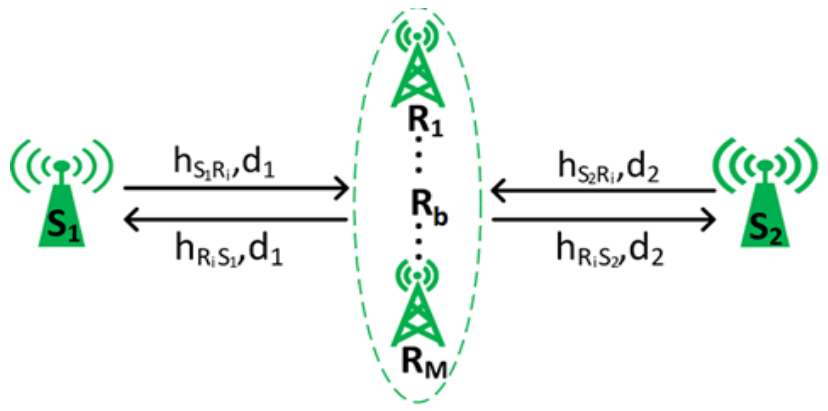

Fig. 1. System model of a two-way relaying NOMA scheme 
In Fig. 1, $\left(h_{S_{1} R_{i}}, d_{1}\right),\left(h_{S_{2} R_{i}}, d_{2}\right),\left(h_{R_{i} S_{1}}, d_{1}\right)$ and $\left(h_{R_{i} S_{2}}, d_{2}\right)$ are Rayleigh fading channel coefficients and normalized distances of links $S_{1}-R_{i}, S_{2}-R_{i}, R_{i}-S_{1}$ and $R_{i}-S_{2}$ respectively. Hence, the random variables (RVs) $g_{S_{k} R_{i}}=\left|h_{S_{k} R_{i}}\right|^{2}$ and $g_{R_{i} S_{k}}=\left|h_{R_{i} S_{k}}\right|^{2}$ have exponential distributions with the same parameter $\lambda_{k}=d_{k}^{\beta}$, where $\beta$ is the path-loss exponent, and $k \in\{1,2\}$. The cumulative distribution function (CDF) and probability density function (pdf) of the RVs $g_{S_{k} R_{i}}$ and $g_{R_{i} S_{k}}$ are expressed as $F_{g_{S_{k} R_{i}}}(x)=F_{g_{R_{i} S_{k}}}(x)=1-e^{-\lambda_{k} x}$ and $f_{g_{S_{k} R_{i}}}(x)=f_{g_{R_{i} S_{k}}}(x)=\lambda_{k} e^{-\lambda_{k} x}$, respectively.

Prior to transmitting packets $x_{1}$ and $x_{2}$, the source node $\mathrm{S}_{1}$ establishes a connection phase to all relays and the source node $S_{2}$ by the media access control (MAC) protocol [2-3]. Firstly, the source nodes $S_{1}$ and $S_{2}$ send in turn to request-to-send (RTS) messages to all relays $R_{i}$, $i \in\{1,2, \ldots M\}$. Next, from receiving the RTS messages, each relay node $\mathrm{R}_{\mathrm{i}}$ can estimate the $h_{S_{k} R_{i}}$, and then broadcasts a helper-ready-to-send (HTS) message which contains the $h_{S_{k} R_{i}}$ to the sources $S_{1}$ and $S_{2}$. After receiving the RTS and HTS messages, the source node $S_{2}$ can estimate the $h_{R_{i} S_{2}}$ and then sends a clear-to-send (CTS) message which comprises these fading channel coefficients. Relying on the reception of the messages HTS and CTS of all relays $\mathrm{R}_{\mathrm{i}}$ and the source $S_{2}$, the source $S_{1}$ can estimate $h_{R_{i} S_{1}}$ and detect the fading channel coefficients $h_{S_{k} R_{i}}$ and $h_{R_{i} S_{2}}$. Hence, the source node $S_{1}$ knows all necessary channel state information to select a best relay $R_{b}$. Finally, the source node $S_{1}$ broadcasts its CTS message to the source node $S_{2}$ and the relays to inform the selected best relay and establish a two-way route from $S_{1}$ to $S_{2}$ and vice versa through that the best relay in the transmission phase.

The operation of the TWDFNOMA protocol occurs in two timeslots as follows. In the first timeslot, the sources $\mathrm{S}_{1}$ and $\mathrm{S}_{2}$ transmit their packets $x_{1}$ and $x_{2}$ to the best-selected relay $\mathrm{R}_{\mathrm{b}}$. In the last timeslot, with knowledge about the channel gains, the best relay $\mathrm{R}_{\mathrm{b}}$ employs the NOMA technology to receive sequentially $x_{1}$ and $x_{2}$, and then mixing these packets $x_{1}$ and $x_{2}$ to create a coded packet $x$ as $x=x_{1} \oplus x_{2}$ (XOR operation in the DNC) before transmitting the packet $x$ back to the sources $S_{1}$ and $S_{2}$.

In this paper, we compare the proposed TWDFNOMA protocol with three protocols studied in [14, 31-32]. The details are discussed as follows. The first protocol in [14], denoted as TWANC, considered the two-timeslot two-way relaying transmission with the ANC solution. The TWANC protocol also operates in two timeslots as the proposed TWDFNOMA protocol but a best relay chosen by making the most of end-to-end SINRs amplifies all received signals at the same time. The second protocol in [31], called as TWDNC, displayed the two-way relaying scheme with the DNC solution and three-timeslot operation. In the first and second timeslots, the source nodes $S_{1}$ and $S_{2}$ broadcast the packets $x_{1}$ and $x_{2}$ to all relays, respectively. In the third timeslot, a best relay selected based on the opportunity relay selection method transmits the mixed packet to the sources $S_{1}$ and $S_{2}$ by using the XOR method as the operation of the proposed TWDFNOMA protocol. The last comparison protocol in [32], denoted as TWNDNC, combines two one-way relaying transmissions to create the two-way relaying transmission between two source nodes. Therefore, the operation procedure of the TWNDNC protocol is considered into four timeslots. In the first and second timeslot, a packet $x_{1}$ is sent from the source $S_{1}$ to the best relay, and from the best relay to the source $S_{2}$. In the third and 
fourth timeslot, a packet $x_{2}$ is transmitted in the opposite direction from the source $S_{2}$ to the source $S_{1}$ through another best relay.

\section{Outage Probability Analysis}

Without loss of generality, we assume that the transmit powers of the sources $S_{1}, S_{2}$ and the relays $R_{i}$ are identical (denoted as $P$ ), and a node successfully decodes the desired packet if its achievable data rate is larger than or equal a target data rate $R_{t}$.

At the first timeslot, the received signal at the relay $R_{i}$ from the sources $S_{1}$ and $S_{2}$ are presented as

$$
y_{R_{i}}=\sqrt{P} h_{S_{1} R_{i}} x_{1}+\sqrt{P} h_{S_{2} R_{i}} x_{2}+n_{R_{i}} \text {, }
$$

where $n_{R_{i}}$ refer to the AWGNs at the relays $\mathrm{R}_{\mathrm{i}}$ with the identical variance $N_{0}$, $E\left\{\left|x_{1}\right|^{2}\right\}=E\left\{\left|x_{2}\right|^{2}\right\}=1$ ( $E\{\chi\}$ is written for the expectation procedure of $\chi$ ).

Based on researches about the NOMA with the SIC in [15-23], in a case $g_{S_{1} R_{i}}>g_{S_{2} R_{i}}$, firstly, the relay $\mathrm{R}_{\mathrm{b}}$ decodes $x_{1}$ in (1), then the component $\sqrt{P} h_{S_{1} R_{i}} x_{1}$ in (1) will be subtracted to decode $x_{2}$. In the first timeslot, the received SINRs $\gamma_{S_{1} R_{i} \mid g_{S_{1} R_{i}}>g_{S_{2} R_{i}}}$ and signal-to-noise ratios (SNRs) $\gamma_{S_{2} R_{i} \mid g_{S_{1} R_{i}}>g_{S_{2} R_{i}}}$ at the relay $\mathrm{R}_{\mathrm{i}}$ for decoding the data $x_{1}$ and $x_{2}$ are obtained, respectively, as follows

$$
\begin{gathered}
\gamma_{S_{1} R_{i} \mid g_{S_{1} R_{i}}>g_{S_{2} R_{i}}}=\frac{P\left|h_{S_{1} R_{i}}\right|^{2}}{P\left|h_{S_{2} R_{i}}\right|^{2}+N_{0}}=\frac{\gamma g_{S_{1} R_{i}}}{\gamma g_{S_{2} R_{i}}+1} . \\
\gamma_{S_{2} R_{i} \mid g_{S_{1} R_{i}}>g_{S_{2} R_{i}}}=\frac{P\left|h_{S_{2} R_{i}}\right|^{2}}{N_{0}}=\gamma g_{S_{2} R_{i}},
\end{gathered}
$$

where $\gamma$ is defined as a transmit SNR, $\gamma=\frac{P}{N_{0}}$.

In the second timeslot, the received signals at the source nodes $\mathrm{S}_{1}$ and $\mathrm{S}_{2}$ are expressed as

$$
y_{S_{j}}=\sqrt{P} h_{R_{i} S_{j}} x+n_{S_{j}},
$$

where $n_{S_{j}}$ refer to the AWGNs at the source nodes $S_{j}$ with the identical variance $N_{0}$. The received SNRs $\gamma_{R_{i} S_{1} \mid g_{S_{1} R_{i}}>g_{S_{2} R_{i}}}$ and $\gamma_{R_{i} S_{2} \mid g_{S_{1} R_{i}}>g_{S_{2} R_{i}}}$ at the source nodes $S_{1}$ and $S_{2}$ for decoding the data $x\left(x=x_{1} \oplus x_{2}\right)$ are solved, respectively, as

$$
\begin{aligned}
\gamma_{R_{i} S_{1} \mid g_{S R_{i}}>g_{S_{2} R_{i}}} & =\frac{P\left|h_{R_{i} S_{1}}\right|^{2}}{N_{0}}=\gamma g_{R_{i} S_{1}} . \\
\gamma_{R_{i} S_{2} \mid g_{S_{1} R_{i}}>g_{S_{2} R_{i}}} & =\frac{P\left|h_{R_{i} S_{2}}\right|^{2}}{N_{0}}=\gamma g_{R_{i} S_{2}} .
\end{aligned}
$$


In the proposed TWDFNOMA protocol, a best relay $R_{b}$ is decided on a criterion as follows

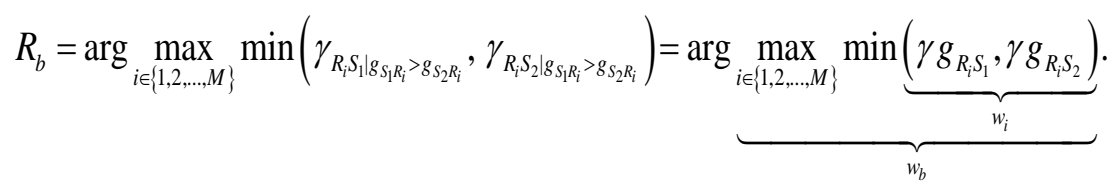

\subsection{The outage probability of the source $S_{1}$ in the case $g_{S_{1} R_{b}}>g_{S_{2} R_{b}}$}

The outage probability of the source $S_{1}$ in the TWDFNOMA protocol occurs when the source $\mathrm{S}_{1}$ does not decode the data packet $\mathrm{x}_{2}$ from the source $\mathrm{S}_{2}$ in the case $g_{S_{1} R_{b}}>g_{S_{2} R_{b}}$, and is expressed as

$$
P_{S_{1} \mid g_{S_{1} R_{b}}>g_{S_{2} R_{b}}}=\underbrace{\operatorname{Pr}\left[R_{S_{2} R_{b} \mid g_{S_{1} R_{b}}>g_{S_{2} R_{b}}}<R_{t}\right]}_{\text {Pr1.1 }}+\underbrace{\operatorname{Pr}\left[R_{S_{2} R_{b} \mid g_{S_{1} R_{b}}>g_{S_{2} R_{b}}} \geq R_{t}, R_{R_{b} S_{1} \mid g_{S_{1} R_{b}}>g_{S_{2} R_{b}}}<R_{t}\right]}_{\text {Pr1.2 }},
$$

where $R_{S_{2} R_{b} \mid g_{S_{1} R_{b}}>g_{S_{2} R_{b}}}$ and $R_{R_{b} S_{1} \mid g_{S_{1} R_{b}}>g_{S_{2} R_{b}}}$ are achievable data rates of connections $\mathrm{S}_{2}-\mathrm{R}_{\mathrm{b}}$ and $\mathrm{R}_{\mathrm{b}}-\mathrm{S}_{1}$, and are obtained as

$$
\begin{aligned}
& R_{S_{2} R_{b} \mid g_{S_{1} R_{b}}>g_{S_{2} R_{b}}}=\frac{1}{2} \log _{2}\left(1+\gamma_{S_{2} R_{b} \mid g_{S_{1} R_{b}}>g_{S_{2} R_{b}}}\right)=\frac{1}{2} \log _{2}\left(1+\gamma g_{S_{2} R_{b}}\right) . \\
& R_{R_{b} S_{1} \mid g_{S_{1} R_{b}}>g_{S_{2} R_{b}}}=\frac{1}{2} \log _{2}\left(1+\gamma_{R_{b} S_{1} \mid g_{S_{1} R_{b}}>g_{S_{2} R_{b}}}\right)=\frac{1}{2} \log _{2}\left(1+\gamma g_{R_{b} S_{1}}\right) .
\end{aligned}
$$

We note that $\gamma_{S_{2} R_{b} \mid g_{S_{1} R_{b}}>g_{S_{2} R_{b}}}$ in (9) and $\gamma_{R_{b} S_{1} \mid g_{S_{1} R_{b}}>g_{S_{2} R_{b}}}$ in (10) are obtained from (3) and (5), respectively.

Base on [32, Eq. (51)], Pr1.1 in (8) is expressed by

$$
\operatorname{Pr} 1.1=\int_{0}^{\infty} \frac{\partial \overbrace{\operatorname{Pr}\left[g_{S_{2} R_{b}}<\theta / \gamma, g_{S_{1} R_{b}}>g_{S_{2} R_{b}}, \min \left(\gamma g_{R_{b} S_{1}}, \gamma g_{R_{b} S_{2}}\right)<x\right]}^{\Omega_{1.1}}}{\partial x} \times \frac{f_{w_{b}}(x)}{f_{w_{i}}(x)} d x,
$$

where $\theta=2^{2 R_{t}}-1$

To solve the Pr1.1 in (11), we use two Lemmas as following.

Lemma 1: A relation of pdf of $\omega_{b}$ and pdf of $\omega_{i}$ is obtained as

Proof: See in Appendix A

$$
\frac{f_{w_{b}}(x)}{f_{w_{i}}(x)}=M\left(1-e^{-\left(\lambda_{1}+\lambda_{2}\right) x / \gamma}\right)^{M-1} \text {. }
$$

Lemma 2: The following expression is valid of $\frac{\partial \Omega_{1.1}}{\partial x}$ :

$$
\frac{\partial \Omega_{1.1}}{\partial x}=\frac{\lambda_{2}}{\gamma}\left(1-e^{-\left(\lambda_{1}+\lambda_{2}\right) \theta / \gamma}\right) e^{-\left(\lambda_{1}+\lambda_{2}\right) x / \gamma}
$$

Proof: Provided in Appendix B.

The exact expression of the outage probability Pr1.1 is provided by the Theorem 1 as

Theorem 1: The probability Pr1.1 is solved by the closed-form expression as

$$
\operatorname{Pr} 1.1=M \lambda_{2}\left(1-e^{-\left(\lambda_{1}+\lambda_{2}\right) \theta / \gamma}\right) \times \frac{1}{\lambda_{1}+\lambda_{2}} \sum_{t-0}^{M-1}(-1)^{t} C_{M-1}^{t} \frac{1}{(t+1)} .
$$


Proof: Substituting Lemma 1 and Lemma 2 into (11), Pr1.1 obtained as

$$
\begin{aligned}
\operatorname{Pr} 1.1 & =\int_{0}^{\infty} \frac{\lambda_{2}}{\gamma}\left(1-e^{-\left(\lambda_{1}+\lambda_{2}\right) \theta / \gamma}\right) e^{-\left(\lambda_{1}+\lambda_{2}\right) x / \gamma} \times M\left(1-e^{-\left(\lambda_{1}+\lambda_{2}\right) x / \gamma}\right)^{M-1} d x \\
& =\frac{M \lambda_{2}}{\gamma}\left(1-e^{-\left(\lambda_{1}+\lambda_{2}\right) \theta / \gamma}\right) \int_{0}^{\infty} e^{-\left(\lambda_{1}+\lambda_{2}\right) x / \gamma} \times\left(1-e^{-\left(\lambda_{1}+\lambda_{2}\right) x / \gamma}\right)^{M-1} d x \\
& =\frac{M \lambda_{2}}{\gamma}\left(1-e^{-\left(\lambda_{1}+\lambda_{2}\right) \theta / \gamma}\right) \times \sum_{t=0}^{M-1}(-1)^{t} C_{M-1}^{t} \int_{0}^{\infty} e^{-\left[\left(\lambda_{1}+\lambda_{2}\right)(t+1)\right] x / \gamma} d x,
\end{aligned}
$$

where $C_{m}^{n}=\frac{(m) !}{n !(m-n) !}$

By solving (15), the Theorem 1 is proven successfully.

Similar as Pr1.1 in (11), the Pr1.2 in (8) is obtained as

$$
\operatorname{Pr}_{1.2}=\int_{0}^{\infty} \frac{\partial \operatorname{Pr}\left[g_{S_{2} R_{b}} \geq \theta / \gamma, g_{S_{1} R_{b}}>g_{S_{2} R_{b}}, \min \left(\gamma g_{R_{b} S_{1}}, \gamma g_{R_{b} S_{2}}\right)<x, g_{R_{b} S_{1}}<\theta / \gamma\right]}{\partial x} \times \frac{f_{w_{b}}(x)}{f_{w_{i}}(x)} d x .
$$

In order to solve the probability Pr1.2 in (16), we also base on Lemma 1 and Lemma 3 where Lemma 3 is presented as follows:

Lemma 3: A derivation of $\Omega_{1.2}$ versus $x$ is given as

$$
\frac{\partial \Omega_{1.2}}{\partial x}= \begin{cases}0 & , x \geq \theta \\ \lambda_{2}\left(\frac{1}{\lambda_{1}+\lambda_{2}} e^{-\left(\lambda_{1}+\lambda_{2}\right) \theta / \gamma}\right)\left(\frac{\left(\lambda_{1}+\lambda_{2}\right)}{\gamma} e^{-\left(\lambda_{1}+\lambda_{2}\right) x / \gamma}-\frac{\lambda_{2}}{\gamma} e^{-\lambda_{1} \theta / \gamma-\lambda_{2} x / \gamma}\right), & x<\theta .\end{cases}
$$

Proof: Given in Appendix C

Theorem 2: A following closed-form expression is valid for the Pr1.2 probability:

$$
\begin{aligned}
\operatorname{Pr} 1.2= & \lambda_{2} M\left(\frac{1}{\lambda_{1}+\lambda_{2}} e^{-\left(\lambda_{1}+\lambda_{2}\right) \theta / \gamma}\right) \\
& \times\left(\begin{array}{l}
\sum_{t=0}^{M-1}(-1)^{t} C_{M-1}^{t} \frac{1}{(t+1)}\left(1-e^{-\left[\left(\lambda_{1}+\lambda_{2}\right)(t+1)\right] \theta / \gamma}\right) \\
-\lambda_{2} e^{-\lambda_{1} \theta / \gamma} \sum_{t=0}^{M-1}(-1)^{t} C_{M-1}^{t} \frac{1}{\left(\lambda_{1}+\lambda_{2}\right) t+\lambda_{2}}\left(1-e^{-\left[\left(\lambda_{1}+\lambda_{2}\right) t+\lambda_{2}\right] \theta / \gamma}\right)
\end{array}\right) .
\end{aligned}
$$

Proof: Substituting Lemma 1 and Lemma 3 into Pr1.2 in (16), the probability Pr1.2 is expressed as 


$$
\begin{aligned}
\operatorname{Pr} 1.2= & \frac{\lambda_{2}}{\gamma} M\left(\frac{1}{\lambda_{1}+\lambda_{2}} e^{-\left(\lambda_{1}+\lambda_{2}\right) \theta / \gamma}\right) \\
& \times \int_{0}^{\theta}\left(\left(\lambda_{1}+\lambda_{2}\right) e^{-\left(\lambda_{1}+\lambda_{2}\right) x / \gamma}-\lambda_{2} e^{-\lambda_{1} \theta / \gamma-\lambda_{2} x / \gamma}\right) \times\left(1-e^{-\left(\lambda_{1}+\lambda_{2}\right) x / \gamma}\right)^{M-1} d x \\
= & \frac{\lambda_{2}}{\gamma} M\left(\frac{1}{\lambda_{1}+\lambda_{2}} e^{-\left(\lambda_{1}+\lambda_{2}\right) \theta / \gamma}\right) \times\left(\begin{array}{l}
\left(\lambda_{1}+\lambda_{2}\right) \sum_{t=0}^{M-1}(-1)^{t} C_{M-1}^{t} \int_{0}^{\theta} e^{-\left[\left(\lambda_{1}+\lambda_{2}\right)(t+1)\right] x / \gamma} d x \\
-\lambda_{2} e^{-\lambda_{1} \theta / \gamma} \sum_{t=}^{M-1}(-1)^{t} C_{M-1}^{t} \int_{0}^{\theta} e^{-\left[\left(\lambda_{1}+\lambda_{2}\right) t+\lambda_{2}\right] x / \gamma} d x
\end{array}\right) .
\end{aligned}
$$

By solving (19) with only two single integrals, we prove the Theorem 2 successfully.

From Theorem 1 in (14) and Theorem 2 in (18), the outage probability $P_{S_{1} \mid g_{S_{1} R_{b}}>g_{S_{2} R_{b}}}=\operatorname{Pr} 1.1+\operatorname{Pr} 1.2$ is solved in the closed-form expression.

\subsection{The outage probability of the source $\mathbf{S}_{2}$ in the case $g_{S_{1} R_{b}}>g_{S_{2} R_{b}}$}

The outage probability of the source $S_{2}$ in the TWDFNOMA protocol occurs when the source $\mathrm{S}_{2}$ does not decode the data $\mathrm{x}_{1}$ from the source $\mathrm{S}_{1}$ with the case $g_{S_{1} R_{b}}>g_{S_{2} R_{b}}$, denoted as $P_{S_{2} \mid g_{S_{1} R_{b}}>g_{S_{2} R_{b}}}$. Similar as the outage probability $P_{S_{1} \mid g_{S_{1} R_{b}}>g_{S_{2} R_{b}}}$ of the source node $\mathrm{S}_{1}$ in the case $g_{S_{1} R_{b}}>g_{S_{2} R_{b}}$ in the section 3.1, the probability $P_{S_{2} \mid g_{S_{1} R_{b}}>g_{S_{2} R_{b}}}$ is achieved as

$$
P_{S_{2} \mid g_{S_{1} R_{b}}>g_{S_{2} R_{b}}}=\underbrace{\operatorname{Pr}\left[R_{S_{1} R_{b} \mid g_{S_{1} R_{b}}>g_{S_{2} R_{b}}}<R_{t}\right]}_{\operatorname{Pr} 2.1}+\underbrace{\operatorname{Pr}\left[R_{S_{1} R_{b} \mid g_{S_{1} R_{b}}>g_{S_{2} R_{b}}} \geq R_{t}, R_{R_{b} S_{2} \mid g_{S_{1} R_{b}}>g_{S_{2} R_{b}}}<R_{t}\right]}_{\operatorname{Pr} 2.2},
$$

where $R_{S_{1} R_{b} \mid g_{S_{1} R_{b}}>g_{S_{2} R_{b}}}$ and $R_{R_{b} S_{2} \mid g_{S_{1} R_{b}}>g_{S_{2} R_{b}}}$ are achievable data rates of connections $\mathrm{S}_{1}-\mathrm{R}_{\mathrm{b}}$ and $\mathrm{R}_{\mathrm{b}}-\mathrm{S}_{2}$, and are related to the received SINRs $\gamma_{S_{1} R_{b} \mid g_{S_{1} R_{b}}>g_{S_{2} R_{b}}}$ and SNR $\gamma_{R_{b} S_{2} \mid g_{S_{1} R_{b}}>g_{S_{2} R_{b}}}$ as

$$
\begin{aligned}
& R_{S_{1} R_{b} \mid g_{S_{1} R_{b}}>g_{S_{2} R_{b}}}=\frac{1}{2} \log _{2}\left(1+\gamma_{S_{1} R_{b} \mid g_{S_{1} R_{b}}>g_{S_{2} R_{b}}}\right)=\frac{1}{2} \log _{2}\left(1+\frac{\gamma g_{S_{1} R_{b}}}{\gamma g_{S_{2} R_{b}}+1}\right) . \\
& R_{R_{b} S_{2} \mid g_{S_{1} R_{b}}>g_{S_{2} R_{b}}}=\frac{1}{2} \log _{2}\left(1+\gamma_{R_{b} S_{2} \mid g_{S_{1} R_{b}}>g_{S_{2} R_{b}}}\right)=\frac{1}{2} \log _{2}\left(1+\gamma g_{R_{b} S_{2}}\right) .
\end{aligned}
$$

As result in [32, Eq. (51)], Pr2.1 is shown as

$$
\operatorname{Pr} 2.1=\int_{0}^{\infty} \frac{\partial \overbrace{\operatorname{Pr}\left[g_{S_{1} R_{b}}>g_{S_{2} R_{b}}, g_{S_{1} R_{b}}<\theta g_{S_{2} R_{b}}+\theta / \gamma, \min \left(\gamma g_{R_{b} S_{1}}, \gamma g_{R_{b} S_{2}}\right)<x\right]}^{\Omega_{2.1}}}{\partial x} \times \frac{f_{w_{b}}(x)}{f_{w_{i}}(x)} d x \text {. }
$$


Lemma 4: A derivation of $\Omega_{2.1}$ versus $x$ is solved as

$$
\frac{\partial \Omega_{2.1}}{\partial x}= \begin{cases}\left(\frac{\lambda_{2}}{\lambda_{1}+\lambda_{2}}-\frac{\lambda_{2} e^{-\lambda_{1} \theta / \gamma}}{\lambda_{2}+\lambda_{1} \theta}\right) \times\left(\frac{\lambda_{1}+\lambda_{2}}{\gamma} e^{-\left(\lambda_{1}+\lambda_{2}\right) x / \gamma}\right) & , 1-\theta \leq 0 \\ \left(\frac{\lambda_{2}}{\lambda_{1}+\lambda_{2}}\left(1-e^{-\left(\lambda_{1}+\lambda_{2}\right) a}\right)-\frac{\lambda_{2}}{\lambda_{2}+\lambda_{1} \theta}\left(e^{-\lambda_{1} \theta / \gamma}-e^{-\lambda_{1} \theta / \gamma-\left(\lambda_{2}+\lambda_{1} \theta\right) a}\right)\right) & , 1-\theta>0\end{cases}
$$

where $a=\frac{\theta}{(1-\theta) \gamma}$.

Proof: as given in Appendix D

Theorem 3: The probability Pr2.1 in (23) is obtained by the closed-form expression in two cases as

-When $\theta \geq 1$ :

$$
\operatorname{Pr} 2.1=\left(\frac{\lambda_{2}}{\lambda_{1}+\lambda_{2}}-\frac{\lambda_{2} e^{-\lambda_{1} \theta / \gamma}}{\lambda_{2}+\lambda_{1} \theta}\right) \times M \sum_{t=0}^{M-1} \frac{(-1)^{t}}{(t+1)} C_{M-1}^{t} .
$$

-When $\theta<1$ :

$$
\operatorname{Pr} 2.1=\left(\frac{\lambda_{2}}{\lambda_{1}+\lambda_{2}}\left(1-e^{-\left(\lambda_{1}+\lambda_{2}\right) a}\right)-\frac{\lambda_{2}}{\lambda_{2}+\lambda_{1} \theta}\left(e^{-\lambda_{1} \theta / \gamma}-e^{-\lambda_{1} \theta / \gamma-\left(\lambda_{2}+\lambda_{1} \theta\right) a}\right)\right) \times M \sum_{t=0}^{M-1} \frac{(-1)^{t}}{(t+1)} C_{M-1}^{t} .
$$

Proof: Substituting Lemma 1 in (12) and Lemma 4 in (24) into (23), Theorem 3 is achieved as

$$
\begin{aligned}
\operatorname{Pr} 2.1 & =\left\{\begin{array}{ll}
\int_{0}^{\infty}\left(\frac{\lambda_{2}}{\lambda_{1}+\lambda_{2}}-\frac{\lambda_{2} e^{-\lambda_{1} \theta / \gamma}}{\lambda_{2}+\lambda_{1} \theta}\right) \times\left(\frac{\lambda_{1}+\lambda_{2}}{\gamma} e^{-\left(\lambda_{1}+\lambda_{2}\right) x / \gamma}\right) \times M\left(1-e^{-\left(\lambda_{1}+\lambda_{2}\right) x / \gamma}\right)^{M-1} d x, & , 1-\theta \leq 0 \\
\int_{0}^{\infty}\left(\frac{\lambda_{2}}{\lambda_{1}+\lambda_{2}}\left(1-e^{-\left(\lambda_{1}+\lambda_{2}\right) a}\right)-\frac{\lambda_{2}}{\lambda_{2}+\lambda_{1} \theta}\left(e^{-\lambda_{1} \theta / \gamma}-e^{-\lambda_{1} \theta / \gamma-\left(\lambda_{2}+\lambda_{1} \theta\right) a}\right)\right) & , 1-\theta>0
\end{array}\right) \\
& = \begin{cases}\left(\frac{\lambda_{1}+\lambda_{2}}{\gamma} e^{-\left(\lambda_{1}+\lambda_{2}\right) x / \gamma}\right) \times M\left(1-e^{-\left(\lambda_{1}+\lambda_{2}\right) x / \gamma}\right)^{M-1} d x \\
\left(\frac{\lambda_{2}}{\lambda_{1}+\lambda_{2}}-\frac{\lambda_{2} e^{-\lambda_{1} \theta / \gamma}}{\lambda_{2}+\lambda_{1} \theta}\right) \times \frac{\lambda_{1}+\lambda_{2}}{\gamma} \times M \sum_{t=0}^{M-1}(-1)^{t} C_{M-1}^{t} \int_{0}^{\infty} e^{-\left(\lambda_{1}+\lambda_{2}\right)(t+1) x / \gamma} d x & , 1-\theta \leq 0 \\
\lambda_{1}+\lambda_{2} & , 1-\theta>0 \\
\left.\times M \sum_{t=0}^{M-1}(-1)^{t} C_{M-1}^{t} \times \frac{\left.\lambda_{1}+\lambda_{2}\right) a}{\gamma}\right)-\frac{\lambda_{2}}{\lambda_{2}+\lambda_{1} \theta} \int_{0}^{\infty} e^{-\left(\lambda_{1}+\lambda_{2}\right)(t+1) x / \gamma} d x & \left.\left., e^{-\lambda_{1} \theta / \gamma}-e^{-\lambda_{1} \theta / \gamma-\left(\lambda_{2}+\lambda_{1} \theta\right) a}\right)\right)\end{cases}
\end{aligned}
$$

By solving (26), the Theorem 3 is obtained successfully. 
Similarly, Pr2.2 is provided as following

$$
\operatorname{Pr} 2.2=\int_{0}^{\infty}\left(\frac{\overbrace{\operatorname{Pr}\left[\begin{array}{l}
g_{S_{1} R_{b}}>g_{S_{2} R_{b}}, g_{S_{1} R_{b}} \geq \theta g_{S_{2} R_{b}}+\theta / \gamma, \\
\min \left(\gamma g_{R_{b} S_{1}}, \gamma g_{R_{b} S_{2}}\right)<x, g_{R_{b} S_{2}}<\theta / \gamma
\end{array}\right]}^{\Omega_{2.2}}}{\partial x}\right) \times \frac{f_{w_{b}}(x)}{f_{w_{i}}(x)} d x .
$$

Lemma 5: A derivation of $\Omega_{2.2}$ versus $x$ is shown in two cases as

-When $x \geq \theta$ :

$$
\frac{\partial \Omega_{2.2}}{\partial x}=0 .
$$

-When $x<\theta$ :

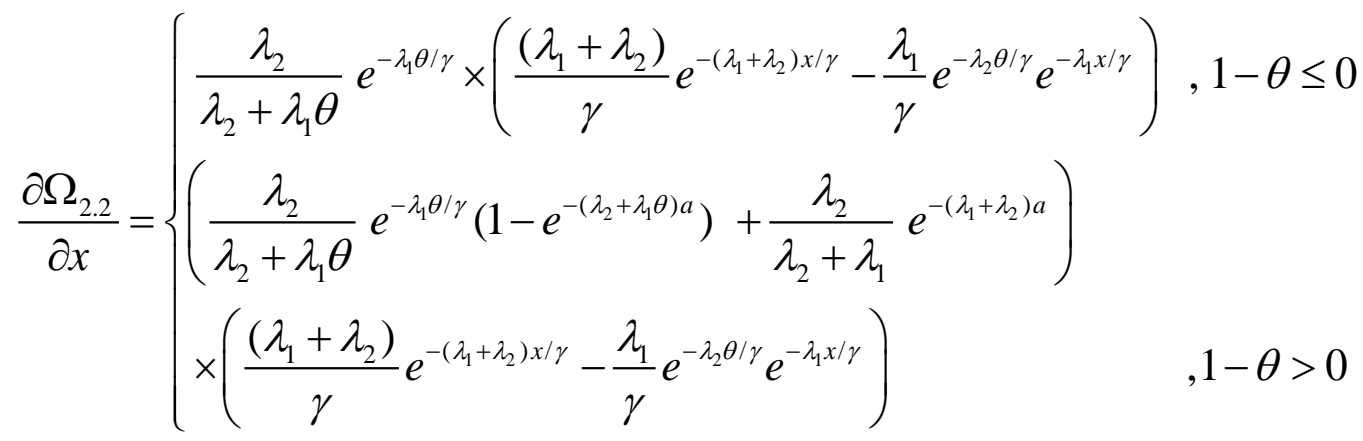

Proof: Given in Appendix E

Theorem 4: The probability $\operatorname{Pr} 2.2$ is obtained by the closed-form expression in two cases as - When $\theta \geq 1$ :

$$
\operatorname{Pr} 2.2=\frac{\lambda_{2}}{\lambda_{2}+\lambda_{1} \theta} e^{-\lambda_{1} \theta / \gamma} \times\left(\begin{array}{l}
M \sum_{t=0}^{M-1}(-1)^{t} C_{M-1}^{t} \frac{1}{(t+1)}\left(1-e^{-\left(\lambda_{1}+\lambda_{2}\right)(t+1) \theta / \gamma}\right) \\
-\lambda_{1} e^{-\lambda_{2} \theta / \gamma} M \sum_{t=0}^{M-1}(-1)^{t} C_{M-1}^{t} \frac{1}{\left(\lambda_{1}+\lambda_{2}\right) t+\lambda_{1}}\left(1-e^{-\left[\left(\lambda_{1}+\lambda_{2}\right) t+\lambda_{1}\right) \theta \theta / \gamma}\right)
\end{array}\right) .
$$

- When $\theta<1$ :

$$
\begin{aligned}
& \operatorname{Pr} 2.2=\left(\frac{\lambda_{2}}{\lambda_{2}+\lambda_{1} \theta} e^{-\lambda_{1} \theta / \gamma}\left(1-e^{-\left(\lambda_{2}+\lambda_{1} \theta\right) a}\right)+\frac{\lambda_{2}}{\lambda_{2}+\lambda_{1}} e^{-\left(\lambda_{1}+\lambda_{2}\right) a}\right) \\
& \times\left(\begin{array}{l}
M \sum_{t=0}^{M-1}(-1)^{t} C_{M-1}^{t} \frac{1}{(t+1)}\left(1-e^{-\left(\lambda_{1}+\lambda_{2}\right)(t+1) \theta / \gamma}\right) \\
-\lambda_{1} e^{-\lambda_{2} \theta / \gamma} M \sum_{t=0}^{M-1}(-1)^{t} C_{M-1}^{t} \frac{1}{\left(\lambda_{1}+\lambda_{2}\right) t+\lambda_{1}}\left(1-e^{-\left[\left(\lambda_{1}+\lambda_{2}\right) t+\lambda_{1}\right) \theta / \gamma}\right)
\end{array}\right) .
\end{aligned}
$$

Proof: Substituting Lemma1 in (12) and Lemma 5 in (29) into (27), Pr2.2 is addressed in two cases $\theta \geq 1$ and $\theta<1$ as 
-For the case $\theta \geq 1$ :

$$
\begin{aligned}
\operatorname{Pr} 2.2=\int_{0}^{\theta}\left(\begin{array}{l}
\frac{\lambda_{2}}{\lambda_{2}+\lambda_{1} \theta} e^{-\lambda_{1} \theta / \gamma} \times\left(\frac{\left(\lambda_{1}+\lambda_{2}\right)}{\gamma} e^{-\left(\lambda_{1}+\lambda_{2}\right) x / \gamma}-\frac{\lambda_{1}}{\gamma} e^{-\lambda_{2} \theta / \gamma} e^{-\lambda_{1} x / \gamma}\right) \\
\times M\left(1-e^{-\left(\lambda_{1}+\lambda_{2}\right) x / \gamma}\right)^{M-1}
\end{array}\right) d x \\
=\frac{\lambda_{2}}{\lambda_{2}+\lambda_{1} \theta} e^{-\lambda_{1} \theta / \gamma} \times\left(\begin{array}{l}
M \frac{\left(\lambda_{1}+\lambda_{2}\right)}{\gamma} \int_{0}^{\theta} e^{-\left(\lambda_{1}+\lambda_{2}\right) x / \gamma}\left(1-e^{-\left(\lambda_{1}+\lambda_{2}\right) x / \gamma}\right)^{M-1} d x \\
-M \frac{\lambda_{1}}{\gamma} e^{-\lambda_{2} \theta / \gamma} \int_{0}^{\theta} e^{-\lambda_{1} x / \gamma}\left(1-e^{-\left(\lambda_{1}+\lambda_{2}\right) x / \gamma}\right)^{M-1} d x
\end{array}\right) .
\end{aligned}
$$

By calculating (31a), Pr2.2 in (30a) is solved successfully.

-For the case $\theta<1$ :

$$
\begin{aligned}
& \operatorname{Pr} 2.2= \int_{0}^{\theta}\left(\left(\frac{\lambda_{2}}{\lambda_{2}+\lambda_{1} \theta} e^{-\lambda_{1} \theta / \gamma}\left(1-e^{-\left(\lambda_{2}+\lambda_{1} \theta\right) a}\right)+\frac{\lambda_{2}}{\lambda_{2}+\lambda_{1}} e^{-\left(\lambda_{1}+\lambda_{2}\right) a}\right)\right. \\
&\left.\times\left(\frac{\left(\lambda_{1}+\lambda_{2}\right)}{\gamma} e^{-\left(\lambda_{1}+\lambda_{2}\right) x / \gamma}-\frac{\lambda_{1}}{\gamma} e^{-\lambda_{2} \theta / \gamma} e^{-\lambda_{1} x / \gamma}\right) \times M\left(1-e^{-\left(\lambda_{1}+\lambda_{2}\right) x / \gamma}\right)^{M-1}\right) d x \\
&=\left(\frac{\lambda_{2}}{\lambda_{2}+\lambda_{1} \theta} e^{-\lambda_{1} \theta / \gamma}\left(1-e^{-\left(\lambda_{2}+\lambda_{1} \theta\right) a}\right)+\frac{\lambda_{2}}{\lambda_{2}+\lambda_{1}} e^{-\left(\lambda_{1}+\lambda_{2}\right) a}\right) \\
& \times\left(\begin{array}{c}
M \frac{\left(\lambda_{1}+\lambda_{2}\right)}{\gamma} \int_{0}^{\theta} e^{-\left(\lambda_{1}+\lambda_{2}\right) x / \gamma}\left(1-e^{-\left(\lambda_{1}+\lambda_{2}\right) x / \gamma}\right)^{M-1} d x \\
-M \frac{\lambda_{1}}{\gamma} e^{-\lambda_{2} \theta / \gamma} \int_{0}^{\theta} e^{-\lambda_{1} x / \gamma}\left(1-e^{-\left(\lambda_{1}+\lambda_{2}\right) x / \gamma}\right)^{M-1} d x
\end{array}\right) .
\end{aligned}
$$

Through solving (31b), Pr2.2 in (30b) is proven successfully.

Hence, from Theorem 3 and Theorem 4, the outage probability $P_{S_{2} \mid g_{S_{1} R_{b}}>g_{S_{2} R_{b}}}=\operatorname{Pr} 2.1+\operatorname{Pr} 2.2$ is calculated in the closed-form expression.

\subsection{The outage probabilities of the sources $S_{1}$ and $S_{2}$ in the remaining case}

$$
g_{S_{2} R_{b}}>g_{S_{1} R_{b}} \text {. }
$$

Because the system model of the proposed TWDFNOMA protocol is symmetric, thus the outage probabilities of the sources $\mathrm{S}_{1}$ and $\mathrm{S}_{2}$ in the remaining case $g_{S_{2} R_{b}}>g_{S_{1} R_{b}}$, denoted as $P_{S_{1} \mid g_{S_{2} R_{b}}>g_{S_{1} R_{b}}}$ and $P_{S_{2} \mid g_{S_{2} R_{b}}>g_{S_{1} R_{b}}}$, are inferred respectively from the outage probabilities $P_{S_{1} \mid g_{S_{1} R_{b}}>g_{S_{2} R_{b}}}$ and $P_{S_{2} \mid g_{S_{1} R_{b}}>g_{S_{2} R_{b}}}$ of the sources $S_{1}$ and $S_{2}$ in the analyzed case $g_{S_{1} R_{b}}>g_{S_{2} R_{b}}$ by changing parameters as $\lambda_{1} \leftrightarrow \lambda_{2}$. 
From the expressions of the $P_{S_{1} \mid g_{S_{1} R_{b}}>g_{S_{2} R_{b}}}$ by (14) and (18), the outage probability $P_{S_{1} \mid g_{S_{2} R_{b}}>g_{S_{1} R_{b}}}$ of the source node $S_{1}$ in the remaining case $g_{S_{2} R_{b}}>g_{S_{1} R_{b}}$ is obtained in the closed-form expressions with two cases as

-When $\theta \geq 1$ :

$$
\begin{aligned}
P_{S_{1} \mid g_{S_{2} R_{b}>}>g_{S_{1} R_{b}}} & \left(\frac{\lambda_{1}}{\lambda_{1}+\lambda_{2}}-\frac{\lambda_{1} e^{-\lambda_{2} \theta / \gamma}}{\lambda_{1}+\lambda_{2} \theta}\right) \times M \sum_{t=0}^{M-1} \frac{(-1)^{t}}{(t+1)} C_{M-1}^{t} \\
& +\frac{\lambda_{1}}{\lambda_{1}+\lambda_{2} \theta} e^{-\lambda_{2} \theta / \gamma} \times\left(\begin{array}{l}
M \sum_{t=0}^{M-1}(-1)^{t} C_{M-1}^{t} \frac{1}{(t+1)}\left(1-e^{-\left(\lambda_{1}+\lambda_{2}\right)(t+1) \theta / \gamma}\right) \\
-\lambda_{2} e^{-\lambda_{1} \theta / \gamma} M \sum_{t=0}^{M-1}(-1)^{t} C_{M-1}^{t} \frac{1}{\left(\lambda_{1}+\lambda_{2}\right) t+\lambda_{2}} \\
\times\left(1-e^{\left.-\left[\left(\lambda_{1}+\lambda_{2}\right) t+\lambda_{2}\right)\right] \theta / \gamma}\right)
\end{array}\right) .
\end{aligned}
$$

-When $\theta<1$ :

$$
\begin{aligned}
& \left(\left(\begin{array}{l}
\frac{\lambda_{1}}{\lambda_{1}+\lambda_{2}}\left(1-e^{-\left(\lambda_{1}+\lambda_{2}\right) a}\right) \\
-\frac{\lambda_{1}}{\lambda_{1}+\lambda_{2} \theta}\left(e^{-\lambda_{2} \theta / \gamma}-e^{-\lambda_{2} \theta / \gamma-\left(\lambda_{1}+\lambda_{2} \theta\right) a}\right)
\end{array}\right) \times M \sum_{t=0}^{M-1} \frac{(-1)^{t}}{(t+1)} C_{M-1}^{t}\right. \\
& P_{S_{1} \mid g_{S_{2} R_{b}>g_{S_{1} R_{b}}}}=\left(+\left(\frac{\lambda_{1}}{\lambda_{1}+\lambda_{2} \theta} e^{-\lambda_{2} \theta / \gamma}\left(1-e^{-\left(\lambda_{1}+\lambda_{2} \theta\right) a}\right)+\frac{\lambda_{1}}{\lambda_{1}+\lambda_{2}} e^{-\left(\lambda_{1}+\lambda_{2}\right) a}\right)\right. \\
& \left.\left(\begin{array}{l}
M \sum_{t=0}^{M-1}(-1)^{t} C_{M-1}^{t} \frac{1}{(t+1)}\left(1-e^{-\left(\lambda_{1}+\lambda_{2}\right)(t+1) \theta / \gamma}\right) \\
-\lambda_{2} e^{-\lambda_{1} \theta / \gamma} M \sum_{t=0}^{M-1}(-1)^{t} C_{M-1}^{t} \frac{1}{\left(\lambda_{1}+\lambda_{2}\right) t+\lambda_{2}}\left(1-e^{\left.-\left[\left(\lambda_{1}+\lambda_{2}\right) t+\lambda_{2}\right)\right] \theta / \gamma}\right)
\end{array}\right)\right)
\end{aligned}
$$

In the same way, the outage probability $P_{S_{2} \mid g_{S_{2} R_{b}}>g_{S_{1} R_{b}}}$ is quickly solved from $P_{S_{2} \mid g_{S_{1} R_{b}}>g_{S_{2} R_{b}}}$ and is obtained in two cases as

-When $\theta \geq 1$ :

$$
\begin{aligned}
& P_{S_{2} \mid g_{S_{2} R_{b}}>g_{S_{1} R_{b}}}=\left(\frac{\lambda_{1}}{\lambda_{1}+\lambda_{2}}-\frac{\lambda_{1} e^{-\lambda_{2} \theta / \gamma}}{\lambda_{1}+\lambda_{2} \theta}\right) \times M \sum_{t=0}^{M-1} \frac{(-1)^{t}}{(t+1)} C_{M-1}^{t} \\
& +\frac{\lambda_{1}}{\lambda_{1}+\lambda_{2} \theta} e^{-\lambda_{2} \theta / \gamma} \times\left(\begin{array}{l}
M \sum_{t=0}^{M-1}(-1)^{t} C_{M-1}^{t} \frac{1}{(t+1)}\left(1-e^{-\left(\lambda_{1}+\lambda_{2}\right)(t+1) \theta / \gamma}\right) \\
-\lambda_{2} e^{-\lambda_{1} \theta / \gamma} M \sum_{t=0}^{M-1}(-1)^{t} C_{M-1}^{t} \frac{1}{\left(\lambda_{1}+\lambda_{2}\right) t+\lambda_{2}}\left(1-e^{\left.-\left[\left(\lambda_{1}+\lambda_{2}\right) t+\lambda_{2}\right)\right] \theta / \gamma}\right)
\end{array}\right) .
\end{aligned}
$$

-When $\theta<1$ : 


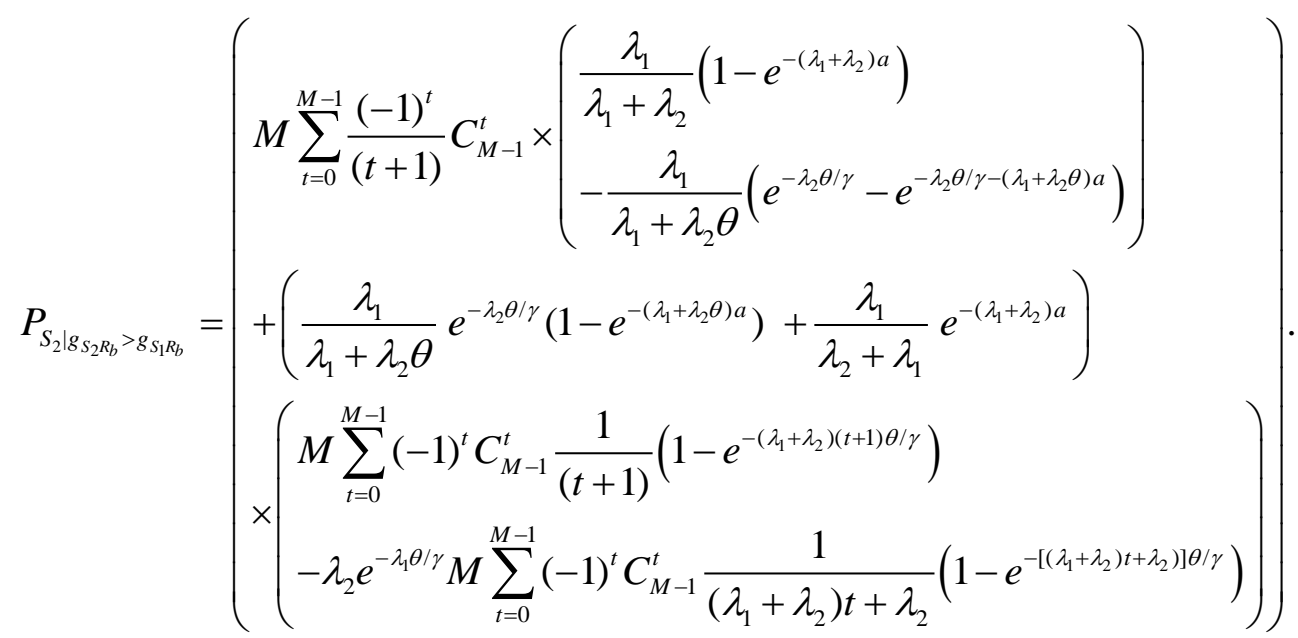

At this time, we have $P_{S_{1} \mid g_{S_{1} R_{b}}>g_{S_{2} R_{b}}}, P_{S_{1} \mid g_{S_{2} R_{b}}>g_{S_{1} R_{b}}}, P_{S_{2} \mid g_{S_{1} R_{b}}>g_{S_{2} R_{b}}}$ and $P_{S_{2} \mid g_{S_{2} R_{b}}>g_{S_{1} R_{b}}}$ on the hand and in order to analyze two-way relaying transmission between the sources $S_{1}$ and $S_{2}$, the sum-outage probability of the proposed TWDFNOMA protocol is inferred as following

$$
P_{T W D F N O M A}^{\text {sum }}=P_{S_{1}}+P_{S_{2}}=P_{S_{1} \mid g_{S_{1} R_{b}}>g_{S_{2} R_{b}}}+P_{S_{1} \mid g_{S_{2} R_{b}}>g_{S_{1} R_{b}}}+P_{S_{2} \mid g_{S_{1} R_{b}}>g_{S_{2} R_{b}}}+P_{S_{2} \mid g_{S_{2} R_{b}}>g_{S_{1} R_{b}}}
$$

\section{Simulation Results}

In this section, we present analysis and simulation results of the outage performances of the proposed TWDFNOMA protocol. These results are also used to compare with the TWDNC protocol [31] , the TWNDNC protocol [32], and the TWANC protocol [14] . The simulation model is considered in the two-dimensional plane with the coordinates as $S_{1}(0,0), S_{2}(1,0)$ and $\mathrm{R}_{\mathrm{i}}(x, y)$, where $0<x<1$ and $i \in\{1,2, \ldots, M\}$. Therefore, $d_{1}=\sqrt{x^{2}+y^{2}}$ and $d_{2}=\sqrt{(1-x)^{2}+y^{2}}$. For fair comparisons, the total energy of the protocols TWDFNOMA, TWDNC, TWNDNC and TWANC are identical, denoted as E. Based on the operation principle of the protocols TWDFNOMA, TWANC, TWDNC and TWNDNC which are described in the section 2, the transmit powers are addressed as $P_{\text {TWDFNOMA }}=P_{T W A N C}=P_{T W D N C}=E / 3 T$, $P_{T W N D N C}=E / 4 T$, where $T$ is the period of a timeslot. The establishment of transmit powers for fair comparisons between these protocols has been considered in [4-5]. Furthermore, the path-loss parameter $\beta$ is set to 3 during simulation operations.

Fig. 2 shows the sum-outage probability of the TWDFNOMA protocol versus $E / N_{0}(d B)$ when the asymmetric network is considered with $x=0.2, y=0$, and $R_{t} \in\{0.5,1\}(\mathrm{bit} / \mathrm{s} / \mathrm{Hz})$. In

Fig. 2, the sum-outage probability of the proposed TWDFNOMA protocol decreases when the $E / N_{o}$ increase. This can be explained by the fact that applying the NOMA technology and the opportunistic relay selection as in formulas (2), (3) and (7), the received SINRs and SNRs at the best relay $R_{b}$, the source nodes $S_{1}$ and $S_{2}$ achieve higher values at large $E / N_{o}$ regions as 
formulas (3), (5) and (6). Hence, the decoding capacities at the nodes $S_{1}, S_{2}$ and $R_{b}$ become better at the larger $E / N_{o}$ regions. Furthermore, the proposed TWDFNOMA protocol with $R_{t}=$ 0.5 (bit/s/Hz) is better than with $R_{t}=1(\mathrm{bit} / \mathrm{s} / \mathrm{Hz})$. Finally, the theoretical analyses are valid by the suitability of the theoretical values and the simulation values.

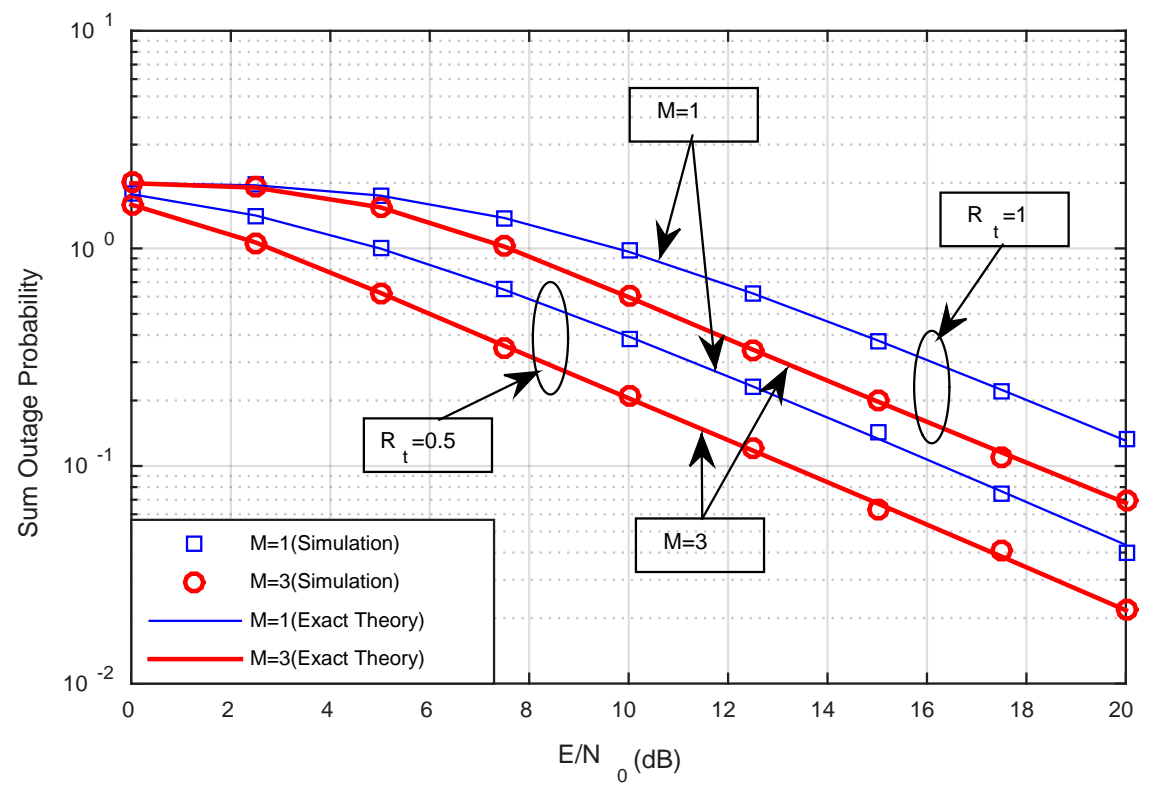

Fig. 2. The sum-outage probability of the proposed TWDFNOMA protocol versus $E / N_{o}(\mathrm{~dB})$ when $x=0.2, y=0, R_{t}$ is considered at 0.5 and $1(\mathrm{bit} / \mathrm{s} / \mathrm{Hz})$

Fig. 3 presents the sum-outage probabilities of the protocols TWDFNOMA, TWANC, TWDNC and TWNDNC versus $E / N_{o}(\mathrm{~dB})$ when $M=3, R_{t}=1(\mathrm{bit} / \mathrm{s} / \mathrm{Hz})$ and the asymmetric network is also considered with $x=0.2, y=0$. From Fig. 3, the sum-outage probabilities decline in increasing $E / N_{o}$ regions for the reason that of high transmit powers. Furthermore, the proposed TWDFNOMA protocol does better than the conventional protocols TWDNC, TWNDNC and TWANC because the proposed TWDFNOMA protocol combines technologies NOMA and DNC to cancel interferences from the stronger signals by the SIC solution and increase the bandwidth utilization efficiency by the XOR operation. We note that all protocols have the same energy for transmitting two signals. 


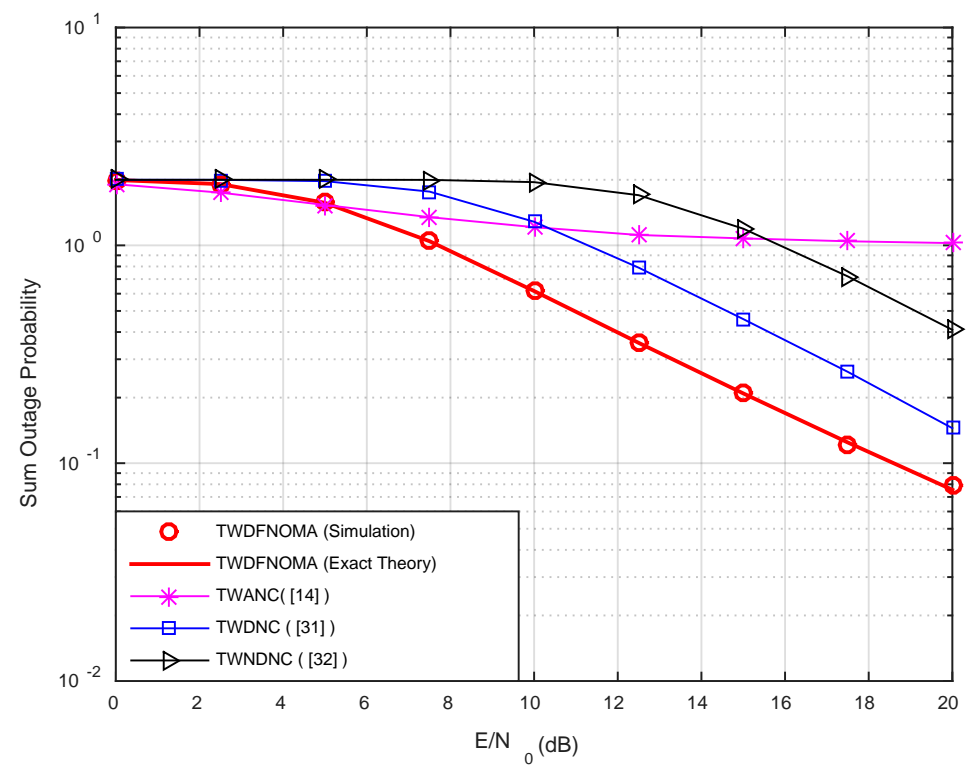

Fig. 3. The sum-outage probabilities of the protocols TWDFNOMA, TWDNC, TWNDNC and TWANC as a function of $E / N_{o}(\mathrm{~dB})$ when $M=3, x=0.2, y=0, R_{t}=1(\mathrm{bit} / \mathrm{s} / \mathrm{Hz})$.

Fig. 4 presents the sum-outage probabilities of the protocols TWDFNOMA, TWANC, TWDNC and TWNDNC as a function of the locations $x$ of the relays on $x$-axis when $y=0.1$, $M=3, E / N_{o}=7(\mathrm{~dB}), R_{t}=1(\mathrm{bit} / \mathrm{s} / \mathrm{Hz})$, and $\mathrm{x}$ is set to move between 0.1 and 0.9 . As shown in Fig. 4, the TWDFNOMA protocol also has the smaller sum-outage probabilities when comparing with the protocols TWDNC, TWNDNC and TWANC. Particularly, the proposed TWDFNOMA protocol achieves best performances at two optimal locations $x=0.3$ and $x=0.7$ whereas the midpoint $x=0.5$ is the optimal location of the TWDNC and TWNDNC protocols. Hence, the NOMA technology is an effective selection for the asymmetric two-way relaying networks, i.e. the optimal locations $x=0.3$ and $x=0.7$ of the cooperative relays.

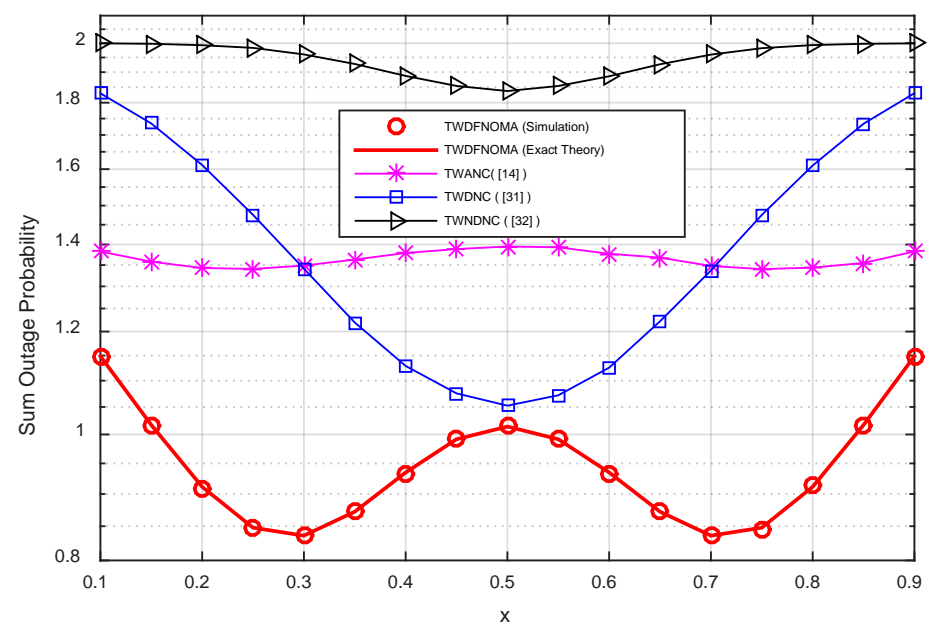

Fig. 4. The sum-outage probabilities of all protocols as a function of the locations $x$ of the relays on $x$-axis, when $M=3, y=0.1, E / N_{o}=7(\mathrm{~dB}), R_{t}=1(\mathrm{bit} / \mathrm{s} / \mathrm{Hz})$. 
Fig. 5 presents the sum-outage probabilities of the protocols TWDFNOMA, TWANC, TWDNC and TWNDNC as a function of the locations $y$ of the relays on $y$-axis when $M=3$, $\mathrm{E} / \mathrm{N}_{\mathrm{o}}=7(\mathrm{~dB}), R_{t}=1(\mathrm{bit} / \mathrm{s} / \mathrm{Hz}), x$ is fixed at $x=0.2$ (the asymmetric two-way relaying networks), and $y$ is set to move between 0.1 and 0.9. In Fig. 5, the proposed TWDFNOMA protocol achieves a better sum-outage performance than the existing protocols TWDNC, TWNDNC and TWANC. It can be seen that when the relays move further on the direction y-axis, the system performance of the protocols TWDFNOMA, TWDNC, TWNDNC and TWANC declines, and then goes towards the worst ranges (about $y>0.9$ ) because of decreasing cooperative operations. All protocols TWDFNOMA, TWANC, TWDNC and TWNDNC perform better when the best relay is set at near locations to the sources $S_{1}$ and $S_{2}(y=0.1)$ whereas $\mathrm{x}$ is fixed to 0.2 .

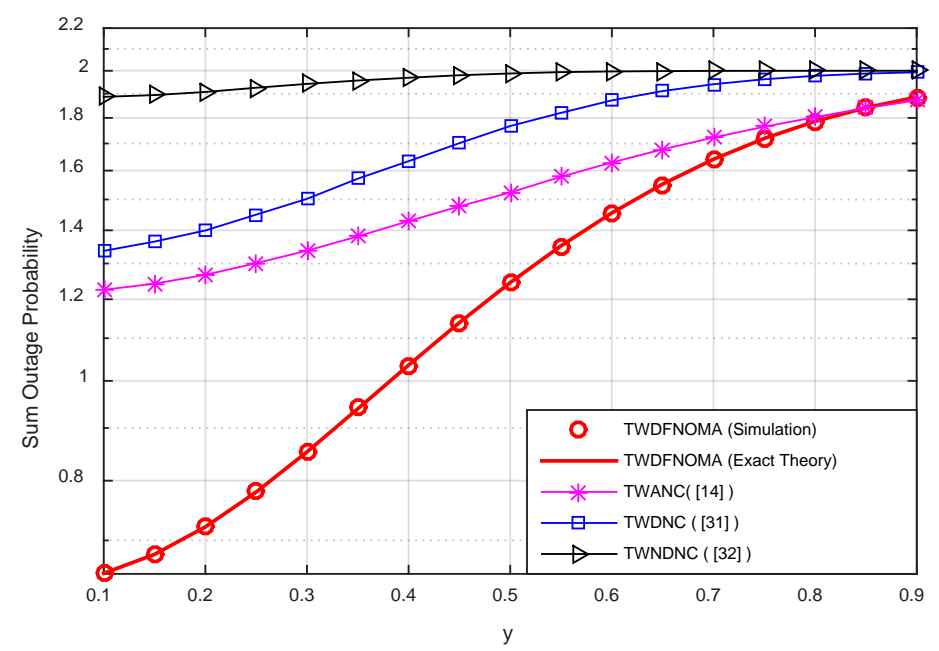

Fig. 5. The sum-outage probabilities of the protocols TWDFNOMA, TWDNC, TWNDNC and TWANC versus the locations y of the relays on $y$-axis when $x=0.2, M=3, E / N_{o}=7(\mathrm{~dB}), R_{t}=1$ (bit/s/Hz).

Fig. 6 and Fig 7 show analysis and simulation results of the protocols TWDFNOMA, TWDNC, TWNDNC and TWANC in which transmit powers of the nodes $S_{1}, S_{2}$ and relays $R_{i}$, $i \in\{1,2, \ldots, M\} \quad$ are set to identical values, denoted as $P_{\text {TWDFNOMA }}=P_{T W A N C}=P_{\text {TWDNC }}=P_{T W N D N C}=P$. The proposed TWDFNOMA protocol and the TWANG protocol use the least number of timeslots with only two timeslots whereas the protocols TWDNC and TWNDNC operate with three and four timeslots, respectively. The evaluations with identical transmit powers and different timeslots have been considered in [24], [32]. Fig. 6 presents the sum-outage probabilities of the protocols TWDFNOMA, TWDNC, TWNDNC and TWANC as versus $P / N_{o}(\mathrm{~dB})$ when $\mathrm{x}=0.2, y=0, \mathrm{M}=3$ and $\mathrm{R}_{\mathrm{t}}=0.5(\mathrm{bit} / \mathrm{s} / \mathrm{Hz})$. As observed from Fig. 6, the sum-outage performances of these protocols decrease when $P / N_{o}$ increase and we can also see that the proposed TWDFNOMA protocol also achieves the smallest sum-outage probabilities. These results prove that the proposed TWDFNOMA protocol gains better performances whereas using the least number of timeslots (two timeslots). It is implied that the proposed TWDFNOMA protocol is essential to enhance the performance of the cooperative two-way scheme. Simulations results verify again the tight accuracy of the derived theoretical analyses. 


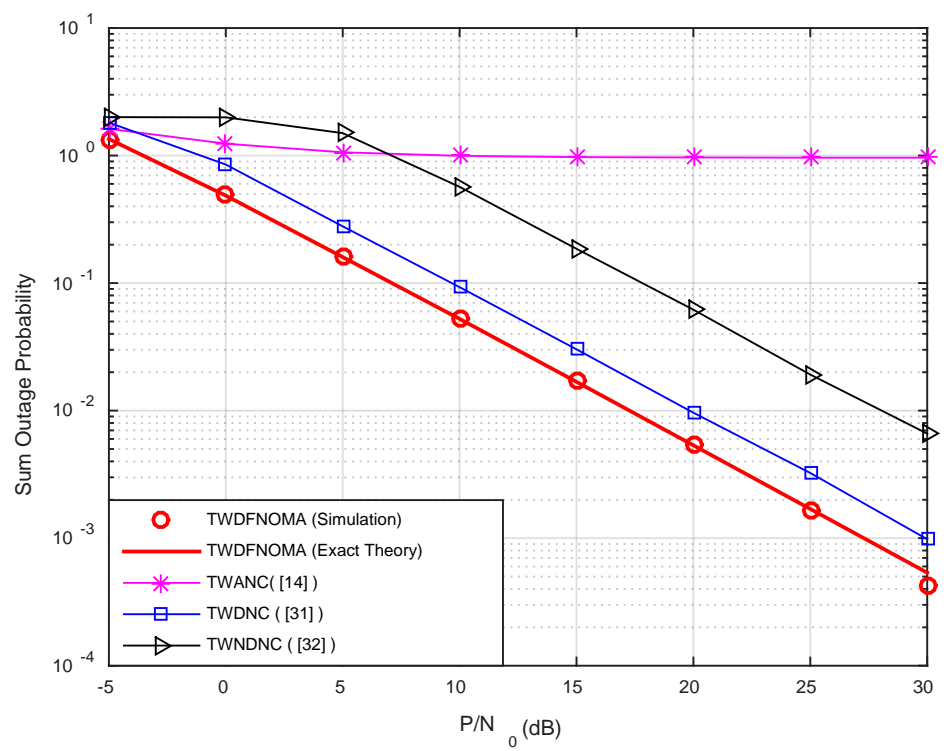

Fig. 6. The sum-outage probabilities of the protocols TWDFNOMA, TWDNC, TWNDNC and TWANC versus $P / N_{o}(\mathrm{~dB})$ when $\mathrm{x}=0.2, y=0, \mathrm{M}=3, \mathrm{R}_{\mathrm{t}}=0.5(\mathrm{bit} / \mathrm{s} / \mathrm{Hz})$.

Fig. 7 shows the sum-outage probabilities of the protocols TWDFNOMA, TWDNC, TWNDNC and TWANC as a function of $R_{t}$ when $x=0.2, \mathrm{y}=0, M=3$ and $P / N_{o}$ is fixed at 10 (dB). From Fig. 7, the rates $R_{t}$ increase, the system performance of all considering protocols decreases. In addition, the proposed TWDFNOMA protocol achieves better performances when comparing with the protocols TWDNC, TWNDNC and TWANC in the condition of identical transmit powers. In this case, we note that the proposed TWDFNOMA protocol only operates in the two timeslots.

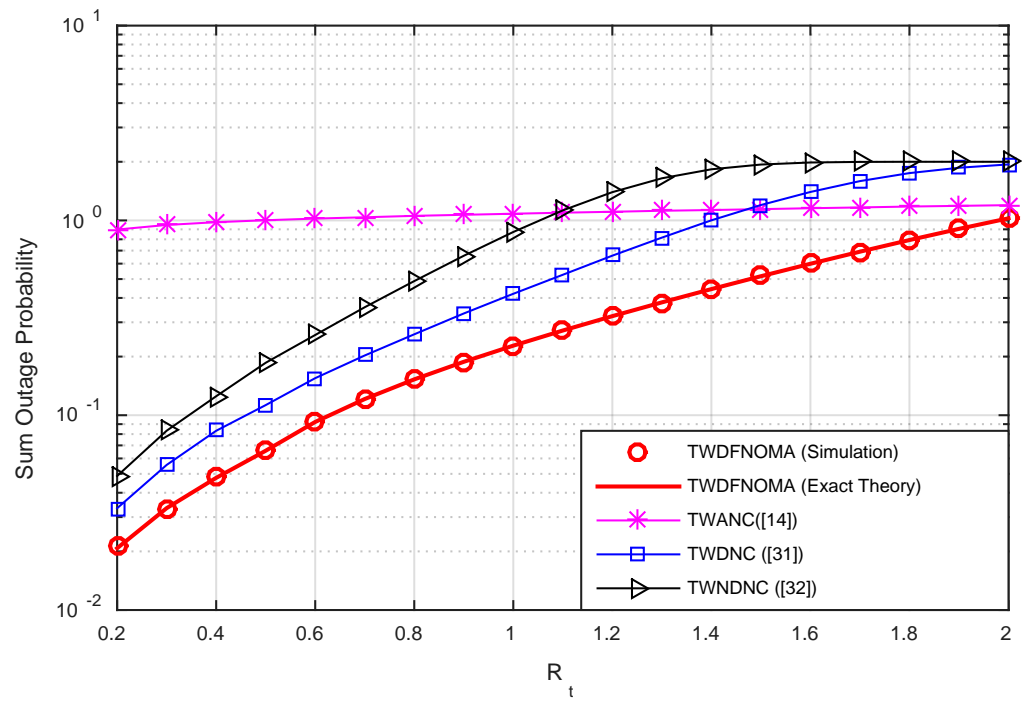

Fig. 7. The sum-outage probabilities of the protocols TWDFNOMA, TWDNC, TWNDNC and TWANC versus $R_{t}$ when $x=0.2, \mathrm{y}=0, M=3, P / N_{o}=10(\mathrm{~dB})$. 


\section{Conclusion}

In this paper, we proposed the two-way relaying scheme with multiple wireless relays in which the best relay is obtained by the opportunistic relay selection method, called as the TWDFNOMA protocol. The best relay applied the SIC to decode the sequence of the received signals and used the DNC solution to encrypt received data from two sources. We analyzed and evaluated the outage performances by the exact closed-form expressions. Simulation and analysis results presented distributions as follows. The proposed TWDFNOMA protocol achieves better performances when compared with the conventional three-timeslot two-way relaying scheme using DNC (denoted as the TWDNC protocol), the four-timeslot two-way relaying scheme without using DNC (denoted as the TWNDNC protocol) and the two-timeslot two-way relaying scheme with AF operations (denoted as the TWANC protocol). The TWDFNOMA protocol reaches the smallest sum-outage probabilities when the cooperative relays form the asymmetric two-way relaying network and are moved to two optimal locations between two source nodes. Furthermore, the proposed TWDFNOMA protocol is improved as the increasing number of relaying nodes. Finally, the outage probability analyses in terms of the closed-form expressions are justified by executing Monte Carlo simulations.

\section{Appendix}

\section{Appendix A: Verification of Lemma 1}

From denotation of $w_{i}$ in (7), the CDF of $w_{i}$ is obtained as

$$
\begin{aligned}
F_{w_{i}}(x) & =\operatorname{Pr}\left(w_{i}<x\right)=\operatorname{Pr}\left[\min \left(\gamma g_{R_{i} S_{1}}, \gamma g_{R_{i} S_{2}}<x\right)\right] \\
& =1-\operatorname{Pr}\left[\min \left(\gamma g_{R_{i} S_{1}}, \gamma g_{R_{i} S_{2}} \geq x\right)\right]=1-\operatorname{Pr}\left(\gamma g_{R_{i} S_{1}} \geq x\right) \times \operatorname{Pr}\left(\gamma g_{R_{i} S_{2}} \geq x\right) \\
& =1-\left(1-F_{g_{R_{i} S_{1}}}(x / \gamma)\right) \times\left(1-F_{g_{R_{i} S_{2}}}(x / \gamma)\right)=1-e^{-\left(\lambda_{1}+\lambda_{2}\right) x / \gamma} .
\end{aligned}
$$

The CDF of $w_{b}$ is given from (7) as

$$
\begin{aligned}
F_{w_{b}}(x) & =\operatorname{Pr}\left(w_{b}<x\right)=\operatorname{Pr}[\underbrace{\max _{n}}_{i=1 \ldots M} w_{i}<x]=\prod_{i=1}^{M} F_{w_{i}}(x) \\
& =\prod_{i=1}^{M}\left(1-e^{-\left(\lambda_{1}+\lambda_{2}\right) x / \gamma}\right)=\left(1-e^{-\left(\lambda_{1}+\lambda_{2}\right) x / \gamma}\right)^{M} .
\end{aligned}
$$

The pdf of $w_{b}$ is inferred as

$$
f_{w_{b}}(x)=\frac{\partial F_{w_{b}}(x)}{\partial x}=M \times\left[1-e^{-\left(\lambda_{1}+\lambda_{2}\right) x / \gamma}\right]^{M-1} \times f_{w_{i}}(x) .
$$

Hence, proof of Lemma 1 is solved completely.

\section{Appendix B: Proof of Lemma 2}

To solve Lemma 2, we calculate $\Omega_{1.1}$ in (11) as follows: 


$$
\begin{aligned}
& \Omega_{1.1}=\operatorname{Pr}\left[g_{S_{2} R_{b}}<\theta / \gamma, g_{S_{1} R_{b}}>g_{S_{2} R_{b}}\right] \times \operatorname{Pr}\left[\min \left(\gamma g_{R_{b} S_{1}}, \gamma g_{R_{b} S_{2}}\right)<x\right] \\
&=\int_{0}^{\theta / \gamma} f_{g_{S_{2} R_{b}}}(y)\left(1-F_{g_{S_{1} R_{b}}}(y)\right) d y \times\left\{\operatorname{Pr}\left[g_{R_{b} S_{1}}<x / \gamma, g_{R_{b} S_{1}}<g_{R_{b} S_{2}}\right]\right. \\
&\left.+\operatorname{Pr}\left[g_{R_{b} S_{2}}<x / \gamma, g_{R_{b} S_{2}}<g_{R_{b} S_{1}}\right]\right\} .
\end{aligned}
$$

Applying the pdf of the RVs $g_{S_{2} R_{b}}, g_{R_{b} S_{1}}$, and $g_{R_{b} S_{2}}$, and the CDF of the RVs $g_{S_{1} R_{b}}$ and $g_{R_{b} S_{1}}$ into (B.1), $\Omega_{1.1}$ is addressed as

$$
\begin{aligned}
\Omega_{1.1} & =\int_{0}^{\theta / y} \lambda_{2} e^{-\lambda_{2} y} e^{-\lambda_{1} y} d y \times\left(\int_{0}^{x / y} \lambda_{1} e^{-\lambda_{1} y} e^{-\lambda_{2} y} d y+\int_{0}^{x / y} \lambda_{2} e^{-\lambda_{2} y} e^{-\lambda_{1} y} d y\right) \\
& =\frac{\lambda_{2}}{\lambda_{1}+\lambda_{2}}\left(1-e^{-\left(\lambda_{1}+\lambda_{2}\right) \theta / \gamma}\right) \times\left(1-e^{-\left(\lambda_{1}+\lambda_{2}\right) x / \gamma}\right) .
\end{aligned}
$$

Performing a derivation of $\Omega_{1.1}$ in (B.2) versus $x$, the proof of Lemma 2 is completed.

\section{Appendix C: Proof of Lemma 3}

From the definition of $\Omega_{1.2}$ in (16), $\Omega_{1.2}$ is calculated as follows:

$$
\begin{aligned}
& \Omega_{1.2}=\operatorname{Pr}\left[g_{S_{2} R_{b}} \geq \theta / \gamma, g_{S_{1} R_{b}}>g_{S_{2} R_{b}}\right] \times \operatorname{Pr}\left[\min \left(\gamma g_{R_{b} S_{1}}, \gamma g_{R_{b} S_{2}}\right)<x, g_{R_{b} S_{1}}<\theta / \gamma\right] \\
& =\left(\operatorname{Pr}\left[g_{R_{b} S_{1}}<\theta / \gamma\right]-\operatorname{Pr}\left[\min \left(\gamma g_{R_{b} S_{1}}, \gamma g_{R_{b} S_{2}}\right)>x, g_{R_{b} S_{1}}<\theta / \gamma\right]\right) \times \int_{\theta / \gamma}^{\infty} f_{g_{S_{2} R_{b}}}(y)\left(1-F_{g_{S_{1} R_{b}}}(y)\right) d y \\
& =\underbrace{\operatorname{Pr}\left[g_{R_{b} S_{1}}<\theta / \gamma\right] \times \int_{\theta / \gamma}^{\infty} f_{g_{S_{2} R_{b}}}(y)\left(1-F_{g_{S_{1} R_{b}}}(y)\right) d y}_{\Omega_{1.21}} \\
& \underbrace{-\operatorname{Pr}\left[x / \gamma<g_{R_{b} S_{1}}<\theta / \gamma\right] \times \operatorname{Pr}\left[g_{R_{b} S_{2}}>x / \gamma\right] \times \int_{\theta / \gamma}^{\infty} f_{g_{S_{2} R_{b}}}(y)\left(1-F_{g_{S 1 R_{b}}}(y)\right) d y .}_{\Omega_{1.22}}
\end{aligned}
$$

Firstly, we calculate the component $\Omega_{1.2 .1}$ in (C.1) as

$$
\Omega_{1.2 .1}=\left(1-e^{-\lambda_{1} \theta / \gamma}\right) \times \int_{\theta / \gamma}^{\infty} \lambda_{2} e^{-\lambda_{2} y} e^{-\lambda_{1} y} d y=\lambda_{2}\left(\frac{1}{\lambda_{1}+\lambda_{2}} e^{-\left(\lambda_{1}+\lambda_{2}\right) \theta / \gamma}\right)\left(1-e^{-\lambda_{1} \theta / \gamma}\right) .
$$

Next, the component $\Omega_{1.2 .2}$ in (C.1) is calculated as

$$
\Omega_{1.2 .2}=-\underbrace{\operatorname{Pr}\left[x / \gamma<g_{R_{b} S_{1}}<\theta / \gamma\right]}_{\Omega_{1.2 .2 .1}} \times \operatorname{Pr}\left[g_{R_{b} S_{2}}>x / \gamma\right] \times \int_{\theta / \gamma}^{\infty} f_{g_{S_{2} R_{b}}}(y)\left(1-F_{g_{S_{1} R_{b}}}(y)\right) d y .
$$

To solve $\Omega_{1.2 .2}$ in (C.3), $\Omega_{1.2 .2 .1}$ need to calculate and the result is obtained as follows: 


$$
\Omega_{1.2 .2 .1}=\left\{\begin{array}{ll}
0 & , x \geq \theta \\
\operatorname{Pr}\left[x / \gamma<g_{R_{b} S_{1}}<\theta / \gamma\right], x<\theta
\end{array}= \begin{cases}0 & , x \geq \theta \\
F_{g_{R_{b} S_{1}}}(\theta / \gamma)-F_{g_{R_{b} S_{1}}}(x / \gamma), & , x<\theta\end{cases}\right.
$$

Substituting (C.4) into (C.3), $\Omega_{1.2 .2}$ is obtained as

$$
\Omega_{1.2 .2}= \begin{cases}0 & , x \geq \theta \\ -\left(F_{g_{R_{b} S_{1}}}(\theta / \gamma)-F_{g_{R_{b} S_{1}}}(x / \gamma)\right) \times \operatorname{Pr}\left[g_{R_{b} S_{2}}>x / \gamma\right] & \\ \times \int_{\theta / \gamma}^{\infty} f_{g_{S_{2} R_{b}}}(y)\left(1-F_{g_{S_{1} R_{b}}}(y)\right) d y & , x<\theta .\end{cases}
$$

Substituting the pdf of the $\mathrm{RV} g_{S_{2} R_{b}}$, and the CDF of the RV $g_{S_{1} R_{b}}$ into (C.5), $\Omega_{1.2 .2}$ is addressed in two cases as

-When $x \geq \theta: \quad \Omega_{1.2 .2}=0$

-When $x<\theta$ :

$$
\begin{aligned}
\Omega_{1.2 .2} & =-\left(e^{-\lambda_{1} x / \gamma}-e^{-\lambda_{1} \theta / \gamma}\right) \times\left(e^{-\lambda_{2} x / \gamma}\right) \times \int_{\theta / \gamma}^{\infty} \lambda_{2} e^{-\lambda_{2} y} e^{-\lambda_{1} y} d y \\
& =-\lambda_{2}\left(\frac{1}{\lambda_{1}+\lambda_{2}} e^{-\left(\lambda_{1}+\lambda_{2}\right) \theta / \gamma}\right)\left(e^{-\left(\lambda_{1}+\lambda_{2}\right) x / \gamma}-e^{-\lambda_{1} \theta / \gamma-\lambda_{2} x / \gamma}\right) .
\end{aligned}
$$

Substituting (C.2) and (C.6) into (C.1), $\Omega_{1.2}$ is obtained as

$$
\Omega_{1.2}=\Omega_{1.2 .1}+\Omega_{1.2 .2}= \begin{cases}\lambda_{2}\left(\frac{1}{\lambda_{1}+\lambda_{2}} e^{-\left(\lambda_{1}+\lambda_{2}\right) \theta / \gamma}\right)\left(1-e^{-\lambda_{1} \theta / \gamma}\right) & , x \geq \theta \\ \lambda_{2}\left(\frac{1}{\lambda_{1}+\lambda_{2}} e^{-\left(\lambda_{1}+\lambda_{2}\right) \theta / \gamma}\right)\left(1-e^{-\lambda_{1} \theta / \gamma}\right) & , x<\theta \\ -\lambda_{2}\left(\frac{1}{\lambda_{1}+\lambda_{2}} e^{-\left(\lambda_{1}+\lambda_{2}\right) \theta / \gamma}\right)\left(e^{-\left(\lambda_{1}+\lambda_{2}\right) x / \gamma}-e^{-\lambda_{1} \theta / \gamma-\lambda_{2} x / \gamma}\right) . & \end{cases}
$$

Performing a derivation of $\Omega_{1.2}$ in (C.7) versus $x$, Lemma 3 is proven completely.

\section{Appendix D: Proof of Lemma 4}

To resolve Lemma 4, we calculate $\Omega_{2.1}$ in (25a) as

$$
\Omega_{2.1}=\overbrace{\operatorname{Pr}\left[g_{S_{2} R_{b}}<g_{S_{1} R_{b}}<\theta g_{S_{2} R_{b}}+\theta / \gamma\right]}^{\phi_{1}} \times \overbrace{\operatorname{Pr}\left[\min \left(\gamma g_{R_{b} S_{1}}, \gamma g_{R_{b} S_{2}}\right)<x\right]}^{\phi_{2}} .
$$

Firstly, $\phi_{1}$ in (D.1) is calculated as follows 


$$
\begin{aligned}
\phi_{1} & =\operatorname{Pr}\left[g_{S_{2} R_{b}}<g_{S_{1} R_{b}}<\theta g_{S_{2} R_{b}}+\theta / \gamma\right] \\
& = \begin{cases}\int_{0}^{\infty} f_{g_{S_{2} R_{b}}}(y)\left(-F_{g_{S_{1} R_{b}}}(x)+F_{g_{S_{1} R_{b}}}(\theta x+\theta / \gamma)\right) d x, \quad 1-\theta \leq 0 \\
\int_{0}^{a} f_{g_{S_{2} R_{b}}}(y)\left(-F_{g_{S_{1} R_{b}}}(x)+F_{g_{S_{1} R_{b}}}(\theta x+\theta / \gamma)\right) d x, & 1-\theta>0 .\end{cases}
\end{aligned}
$$

where $a=\frac{\theta}{(1-\theta) \gamma}$.

Applying the pdf of the RV $g_{S_{2} R_{b}}$ and the CDF of the RV $g_{S_{1} R_{b}}$ into (D.2), $\phi_{1}$ is resolved as

$$
\phi_{1}=\left\{\begin{array}{l}
\int_{0}^{\infty} \lambda_{2} e^{-\lambda_{2} x}\left(e^{-\lambda_{1} x}-e^{-\lambda_{1}(\theta x+\theta / \gamma)}\right) d x=\frac{\lambda_{2}}{\lambda_{1}+\lambda_{2}}-\frac{\lambda_{2} e^{-\lambda_{1} \theta / \gamma}}{\lambda_{2}+\lambda_{1} \theta} \quad, 1-\theta \leq 0 \\
\int_{0}^{a} \lambda_{2} e^{-\lambda_{2} x}\left(e^{-\lambda_{1} x}-e^{-\lambda_{1}(\theta x+\theta / \gamma)}\right) d x \\
=\frac{\lambda_{2}}{\lambda_{1}+\lambda_{2}}\left(1-e^{-\left(\lambda_{1}+\lambda_{2}\right) a}\right)-\frac{\lambda_{2}}{\lambda_{2}+\lambda_{1} \theta}\left(e^{-\lambda_{1} \theta / \gamma}-e^{-\lambda_{1} \theta / \gamma-\left(\lambda_{2}+\lambda_{1} \theta\right) a}\right), 1-\theta>0 .
\end{array}\right.
$$

Next, $\phi_{2}$ in (D.1) is obtained as

$$
\begin{aligned}
\phi_{2} & =\operatorname{Pr}\left[\min \left(\gamma g_{R_{b} S_{1}}, \gamma g_{R_{b} S_{2}}\right)<x\right]=1-\operatorname{Pr}\left[\min \left(\gamma g_{R_{b} S_{1}}, \gamma g_{R_{b} S_{2}}\right) \geq x\right] \\
& =1-\operatorname{Pr}\left[\gamma g_{R_{b} S_{1}} \geq x\right] \times \operatorname{Pr}\left[\gamma g_{R_{b} S_{2}} \geq x\right]=1-\left(1-F_{g_{R_{b} S_{1}}}(x / \gamma)\right) \times\left(1-F_{R_{b} S_{2}}(x / \gamma)\right. \\
& =1-e^{-\left(\lambda_{1}+\lambda_{2}\right) x / \gamma} .
\end{aligned}
$$

Substituting (D.2) and (D.4) into (D.1), $\Omega_{2.1}$ is obtained as follows:

$$
\Omega_{2.1}= \begin{cases}\left(\frac{\lambda_{2}}{\lambda_{1}+\lambda_{2}}-\frac{\lambda_{2} e^{-\lambda_{1} \theta / \gamma}}{\lambda_{2}+\lambda_{1} \theta}\right) \times\left(1-e^{-\left(\lambda_{1}+\lambda_{2}\right) \times / \gamma}\right) & , 1-\theta \leq 0 \\ \left(\frac{\lambda_{2}}{\lambda_{1}+\lambda_{2}}\left(1-e^{-\left(\lambda_{1}+\lambda_{2}\right) a}\right)-\frac{\lambda_{2}}{\lambda_{2}+\lambda_{1} \theta}\left(e^{-\lambda_{1} \theta / \gamma}-e^{-\lambda_{1} \theta / \gamma-\left(\lambda_{2}+\lambda_{1} \theta\right) a}\right)\right) & \\ \times\left(1-e^{-\left(\lambda_{1}+\lambda_{2}\right) x / \gamma}\right) & , 1-\theta>0 .\end{cases}
$$

Performing a derivation of $\Omega_{2.1}$ in (D.5) versus $x$, the proof of the Lemma 4 is solved completely.

\section{Appendix E: Proof of Lemma 5}

To resolve Lemma 5, we express $\Omega_{2.2}$ in (27) as 


$$
\begin{aligned}
\Omega_{2.2}= & \overbrace{\operatorname{Pr}\left[g_{S_{1} R_{b}}>\right.} g_{S_{2} R_{b}}, g_{S_{1} R_{b}} \geq \theta g_{S_{2} R_{b}}+\theta / \gamma] \\
& \times \overbrace{\operatorname{Pr}\left[\min \left(\gamma g_{R_{b} S_{1}}, \gamma g_{R_{b} S_{2}}\right)<x, g_{R_{b} S_{2}}<\theta / \gamma\right]}^{\phi_{3}} .
\end{aligned}
$$

Firstly, $\phi_{3}$ in (E.1) is calculated as

$$
\begin{aligned}
& \phi_{3}= \begin{cases}\operatorname{Pr}\left[g_{S_{1} R_{b}} \geq \theta g_{S_{2} R_{b}}+\theta / \gamma\right] & , 1-\theta \leq 0 \\
\left.\begin{array}{ll}
\operatorname{Pr}\left[g_{S_{1} R_{b}} \geq \theta g_{S_{2} R_{b}}+\theta / \gamma, g_{S_{2} R_{b}}<\theta g_{S_{1} R_{b}}+\theta / \gamma\right] \\
+\operatorname{Pr}\left[g_{S_{1} R_{b}}>g_{S_{2} R_{b}}, g_{S_{2} R_{b}} \geq \theta g_{S_{2} R_{b}}+\theta / \gamma\right]
\end{array}\right) & 1-\theta>0\end{cases} \\
& \int_{0}^{\infty} f_{g_{S_{2} R_{b}}}(y)\left(1-F_{g_{S_{1} R_{b}}}(\theta y+\theta / \gamma) d y \quad, 1-\theta \leq 0\right. \\
& = \begin{cases}\int_{0}^{a} f_{g_{S_{2} R_{b}}}(y)\left(1-F_{g_{S_{1} R_{b}}}(\theta y+\theta / \gamma)\right) d y & , 1-\theta>0 . \\
& \end{cases} \\
& +\int_{a}^{\infty} f_{g_{S_{2} R_{b}}}(y)\left(1-F_{g_{S_{1} R_{b}}}(y)\right) d y
\end{aligned}
$$

Applying the pdf of the RV $g_{S_{2} R_{b}}$ and the CDF of the RV $g_{S_{1} R_{b}}$ into (E.2), $\phi_{3}$ is addressed as

$$
= \begin{cases}\int_{0}^{\infty} \lambda_{2} e^{-\lambda_{2} y} e^{-\lambda_{1}(\theta y+\theta / \gamma)} d y=\frac{\lambda_{2}}{\lambda_{2}+\lambda_{1} \theta} e^{-\lambda_{1} \theta / \gamma} & , 1-\theta \leq 0 \\ \int_{0}^{a} \lambda_{2} e^{-\lambda_{2} y} e^{-\lambda_{1}(\theta y+\theta / \gamma)} d y+\int_{a}^{\infty} \lambda_{2} e^{-\lambda_{2} y} e^{-\lambda_{1} y} d y & \\ =\frac{\lambda_{2}}{\lambda_{2}+\lambda_{1} \theta} e^{-\lambda_{1} \theta / \gamma}\left(1-e^{-\left(\lambda_{2}+\lambda_{1} \theta\right) a}\right)+\frac{\lambda_{2}}{\lambda_{2}+\lambda_{1}} e^{-\left(\lambda_{1}+\lambda_{2}\right) a}, & 1-\theta>0 .\end{cases}
$$

Similarly, $\phi_{4}$ in (E.1) is solved as following

$$
\begin{aligned}
\phi_{4} & =\operatorname{Pr}\left[g_{R_{b} S_{2}}<\theta / \gamma\right]-\operatorname{Pr}\left[\min \left(\gamma g_{R_{b} S_{1}}, \gamma g_{R_{b} S_{2}}\right)>x, g_{R_{b} S_{2}}<\theta / \gamma\right] \\
& =\operatorname{Pr}\left[g_{R_{b} S_{2}}<\theta / \gamma\right]-\operatorname{Pr}\left[x / \gamma<g_{R_{b} S_{2}}<\theta / \gamma\right] \times \operatorname{Pr}\left[g_{R_{b} S_{1}}>x / \gamma\right] \\
& = \begin{cases}\left(1-e^{-\lambda_{2} \theta / \gamma}\right)-\left(e^{-\lambda_{2} x / \gamma}-e^{-\lambda_{2} \theta / \gamma}\right) \times\left(e^{-\lambda_{1} x / \gamma}\right) & , x<\theta \\
\left(1-e^{-\lambda_{2} \theta / \gamma}\right) & , x \geq \theta .\end{cases}
\end{aligned}
$$

From (E3) and (E4), it is easy to show that when $x \geq \theta, \Omega_{2.2}$ is not a function of $x$, then derivation of $\Omega_{2.2}$ versus $x$ equals to 0 .

-When $x<\theta$, substituting (E.3) and (E.4) into (E.1), $\Omega_{2.2}$ is obtained as follows: 


$$
\Omega_{2.2}= \begin{cases}\frac{\lambda_{2}}{\lambda_{2}+\lambda_{1} \theta} e^{-\lambda_{1} \theta / \gamma} \times\left[\left(1-e^{-\lambda_{2} \theta / \gamma}\right)-\left(e^{-\lambda_{2} x / \gamma}-e^{-\lambda_{2} \theta / \gamma}\right) \times\left(e^{-\lambda_{1} x / \gamma}\right)\right] & , \theta \geq 1 \\ {\left[\frac{\lambda_{2}}{\lambda_{2}+\lambda_{1} \theta} e^{-\lambda_{1} \theta / \gamma}\left(1-e^{-\left(\lambda_{2}+\lambda_{1} \theta\right) a}\right)+\frac{\lambda_{2}}{\lambda_{2}+\lambda_{1}} e^{-\left(\lambda_{1}+\lambda_{2}\right) a}\right]} & , \theta<1 \\ \times\left[\left(1-e^{-\lambda_{2} \theta / \gamma}\right)-\left(e^{-\lambda_{2} x / \gamma}-e^{-\lambda_{2} \theta / \gamma}\right) \times\left(e^{-\lambda_{1} x / \gamma}\right)\right] & , \theta 00 \text { of }\end{cases}
$$

Performing a derivation of $\Omega_{2.2}$ in (E.5) versus $x$, we solved the proof of the Lemma 5 successfully.

\section{Acknowledgments}

This work was supported by the grant SGS reg. No. SP2019/41 conducted at VSB Technical University of Ostrava, Czech Republic and was partially funded by Ho Chi Minh City University of Technology and Education under grant number T2019-51TĐ.

\section{References}

[1] L. Zhang, J. Liu, M. Xiao, G. Wu, Y. C. Liang and S. Li, "Performance Analysis and Optimization in Downlink NOMA Systems With Cooperative Full-Duplex Relaying," IEEE Journal on Selected Areas in Communications, vol. 35, no. 10, pp. 2398-2412, Oct. 2017. Article (CrossRef Link)

[2] L. Pei, T. Zhifeng, L. Zinan, E. Erkip and S. Panwar, "Cooperative wireless communications: a cross-layer approach,” IEEE on Wireless Communications, vol. 13, no. 4, pp. 84-92, Aug. 2006. Article (CrossRef Link)

[3] P. N. Son and H. Y. Kong, "Exact outage analysis of Energy Harvesting Underlay Cooperative Cognitive Networks," IEICE Transactions on Communications, vol. E98-B, no. 4, pp. 661-672, Apr. 2015. Article (CrossRef Link)

[4] M. Ju, I. Kim and D. I. Kim, "Joint Relay Selection and Relay Ordering for DF-Based Cooperative Relay Networks," IEEE Transactions on Communications, vol. 60, no. 4, pp. 908-915, April 2012. Article (CrossRef Link)

[5] P. N. Son and H. Y. Kong, "Performance Analysis of Decode-and-Forward Scheme with Relay Ordering for Secondary Spectrum Access," Wireless Personal Communications (WPC), vol. 79, no. 1, pp. 85-103, Nov. 2014. Article (CrossRef Link)

[6] R. Jiao, L. Dai, J. Zhang, R. MacKenzie, and M. Hao, "On the Performance of NOMA-Based Cooperative Relaying Systems over Rician Fading Channels," IEEE Transactions on Vehicular Technology, vol. 66, no.12, pp.11409-11413, Jul. 2017. Article (CrossRef Link)

[7] K. H. Liu, "Performance Analysis of Relay Selection for Cooperative Relays Based on Wireless Power Transfer With Finite Energy Storage," IEEE Transactions on Vehicular Technology, vol. 65, pp. 5110-5121, 2016. Article (CrossRef Link)

[8] K. Senthil Kumar and R. Amutha, "An Algorithm for Energy Efficient Cooperative Communication in Wireless Sensor Networks," KSII Transactions on Internet and Information Systems, vol. 10, no. 7, pp. 3080-3099, 2016. Article (CrossRef Link)

[9] F. Wang, S. Guo, Y. Yang and B. Xiao, "Relay Selection and Power Allocation for Cooperative Communication Networks With Energy Harvesting," IEEE Systems Journal, vol. 12, vol. 1, pp. 735 - 746, March 2018. Article (CrossRef Link) 
[10] S. Touati, H. Boujemaa, M. A. Al Hussain, F. Alturki and N. Abed, "Cooperative HARQ protocols using semi-blind relays for underlay cognitive radio networks," Telecommunication Systems, vol. 63, no. 2, pp. 287-295, Oct. 2016. Article (CrossRef Link)

[11] D. Li, “Amplify-and-Forward Relay Sharing for Both Primary and Cognitive Users," IEEE Transactions on Vehicular Technology, Vol. 65, no. 4, p. 2796-2801, Apr. 2016. Article (CrossRef Link)

[12] C. Zhang, J. Ge, J. Li, Y. Rui and M. Guizani, "A Unified Approach for Calculating the Outage Performance of Two-Way AF Relaying Over Fading Channels," IEEE Transactions on Vehicular Technology, vol. 64, no. 3, pp. 1218-1229, Mar. 2015. Article (CrossRef Link)

[13] Jianrong Bao, Bin Jiang, Chao Liu, Xianyang Jiang and Minhong Sun, “Optimized Relay Selection and Power Allocation by an Exclusive Method in Multi-Relay AF Cooperative Networks,” KSII Transactions on Internet and Information Systems, vol. 11, no. 7, pp. 3524-3542, 2017. Article (CrossRef Link)

[14] L. Song, "Relay Selection for Two-Way Relaying With Amplify-and-Forward Protocols," IEEE Transactions on Vehicular Technology, vol. 60, no. 4, pp. 1954-1959, May 2011. Article (CrossRef Link)

[15] J. B. Kim and I. H. Lee, "Capacity Analysis of Cooperative Relaying Systems Using Non-Orthogonal Multiple Access," IEEE Communications Letters, vol. 19, no. 11, pp. 1949-1952, Nov. 2015. Article (CrossRef Link)

[16] Z. Ding, M. Peng and H. V. Poor, "Cooperative Non-Orthogonal Multiple Access in 5G Systems," IEEE Communications Letters, vol. 19, no. 8, pp. 1462-1465, Aug. 2015. Article (CrossRef Link)

[17] H. Chingoska, Z. Hadzi-Velkov, I. Nikoloska and N. Zlatanov, "Resource Allocation in Wireless Powered Communication Networks With Non-Orthogonal Multiple Access," IEEE Wireless Communications Letters, vol. 5, no. 6, pp. 684-687, Dec. 2016. Article (CrossRef Link)

[18] S. Lee, D. Benevides da Costa and T. Q. Duong, "Outage probability of non-orthogonal multiple access schemes with partial relay selection," in Proc. of IEEE 27th Annual International Symposium on Personal, Indoor, and Mobile Radio Communications (PIMRC), pp. 1-6, Sep. 2016. Article (CrossRef Link)

[19] Y. Liu, G. Pan, H. Zhang and M. Song, "Hybrid Decode-Forward \& Amplify-Forward Relaying With Non-Orthogonal Multiple Access," IEEE Access, vol. 4, pp. 4912-4921, 2016. Article (CrossRef Link)

[20] W. Duan, M. Wen, Z. Xiong and M. H. Lee, "Two-Stage Power Allocation for Dual-Hop Relaying Systems With Non-Orthogonal Multiple Access," IEEE Access, vol. 5, pp. 2254-2261, 2017. Article (CrossRef Link)

[21] J. Zhu, J. Wang, Y. Huang, S. He, X. You and L. Yang, "On Optimal Power Allocation for Downlink Non-Orthogonal Multiple Access Systems," IEEE Journal on Selected Areas in Communications, vol. 35, no. 12, pp. 2744 - 2757, Dec. 2017. Article (CrossRef Link)

[22] L. Lv, J. Chen, Q. Ni and Z. Ding, "Design of Cooperative Non-Orthogonal Multicast Cognitive Multiple Access for 5G Systems: User Scheduling and Performance Analysis," IEEE Transactions on Communications, vol. 65, no. 6, pp. 2641-2656, June 2017. Article (CrossRef Link)

[23] Peng Lan, Lizhen Chenl, Guowei Zhang and Fenggang Sun, “Optimal Power Allocation and Relay Selection for Cognitive Relay Networks using Non-orthogonal Cooperative Protocol,” KSII Transactions on Internet and Information Systems, vol. 10, no. 5, pp. 2047-2066, 2016. Article (CrossRef Link)

[24] T. T Duy and H. Y Kong, "Exact outage probability of cognitive two-way relaying scheme with opportunistic relay selection under interference constraint,” IET Communications, vol. 6, no. 16, pp. 2750-2759, Nov. 2012. Article (CrossRef Link)

[25] F. Fan, X. Lei, H. Chen and W. Zhou, "Impact of channel estimation error on fixed-gain two-way relay network with user/antenna selection,” Transactions on Emerging Telecommunications Technologies, vol. 25, no. 5, pp. 490-495, 2014. Article (CrossRef Link) 
[26] P. Popovski and H. Yomo, "Physical network coding in two-way wireless relay channels," in Proc. of IEEE International Conference on Communications, pp. 707-712, Aug. 2007. Article (CrossRef Link)

[27] A. Sheikh and A. Olfat, "New Beamforming and Relay Selection for Two-Way Decode-and-Forward Relay Networks," IEEE Transactions on Vehicular Technology, vol. 65, no. 3, pp. 1354-1366, March 2016. Article (CrossRef Link)

[28] Q.F. Zhou, W.H. Mow, S. Zhang and D. Toumpakaris, “Two-Way Decode-and-Forward for Low-Complexity Wireless Relaying: Selective Forwarding Versus One-Bit Soft Forwarding," IEEE Transactions on Wireless, vol. 15, no. 3, p. 1866-1880, Mar. 2016. Article (CrossRef Link)

[29] C. Peng, F. Li and H. Liu, "Optimal Power Splitting in Two-Way Decode-and-Forward Relay Networks," IEEE Communications Letters, vol. 21, no. 9, pp. 2009-2012, Sept. 2017. Article (CrossRef Link)

[30] R. Cao, H. Gao, T. Lv, S. Yang and S. Huang, "Phase-Rotation-Aided Relay Selection in Two-Way Decode-and-Forward Relay Networks," IEEE Transactions on Vehicular Technology, vol. 65, no. 5, pp. 2922-2935, May 2016. Article (CrossRef Link)

[31] Q. F. Zhou, Y. Li, F. C. M. Lau and B. Vucetic, "Decode-and-Forward Two-Way Relaying with Network Coding and Opportunistic Relay Selection," IEEE Transactions on Communications, vol. 58, no. 11, pp. 3070-3076, November 2010. Article (CrossRef Link)

[32] P. N. Son and H. Y. Kong, "Exact outage probability of two-way decode-and-forward scheme with opportunistic relay selection under physical layer security," Wireless personal communications, vol. 77, no. 4, pp. 2889-2917, 2014. Article (CrossRef Link)

[33] P. N. Son and H. Y. Kong, "Improvement of the two-way decode-and-forward scheme by energy harvesting and digital network coding relay," Transactions on Emerging Telecommunications Technologies (ETT), vol. 28, no. 3, pp. 1-14, March 2017. Article (CrossRef Link)

[34] X. Wang, M. Jia, I. W.-H. Ho, Q. Guo and F. C. M. Lau, "Exploiting fullduplex two-Way relay cooperative non-orthogonal multiple access,” IEEE Transactions on Communications, vol. 67, no. 4, pp. 2716-2729, April 2019. Article (CrossRef Link)

[35] X. Yue, Y. Liu, S. Kang, A. Nallanathan and Y. Chen, "Modeling and analysis of two-way relay non-orthogonal multiple access systems," IEEE Transactions on Communications, vol. 66, no. 9, pp. 3784-3796, Sept. 2018. Article (CrossRef Link) 


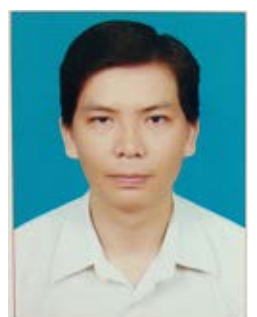

Tan-Phuoc Huynh received the M.Sc. from University of Information Technology VietNam National University Ho Chi Minh in 2008. He received the Ph.D. degree in Communication Technology from Technical University of Ostrava, Czech Republic, in 2019. He is currently a Lecturer at the School of Engineering, Eastern International University, Binh Duong City. His major research interests are wireless communication in 5G, and non-orthogonal multiple access. energy harvesting, performance of cognitive radio and network security.

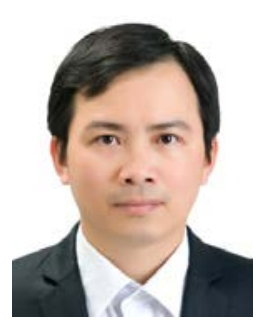

Pham Ngoc Son received the B.E. degree (2005) and M.Eng. degree (2009) in Electronics and Telecommunications Engineering from Post and Telecommunication Institute of Technology, Ho Chi Minh City and Ho Chi Minh City University of Technology, Vietnam, respectively. In 2015, he received the Ph.D. degree in Electrical Engineering from University of Ulsan, South Korea. He is currently a Lecturer in the Faculty of Electrical and Electronics Engineering of Ho Chi Minh City University of Technology and Education (HCMUTE). His major research interests are cooperative communication, cognitive radio, physical layer security, energy harvesting and non-orthogonal multiple access.

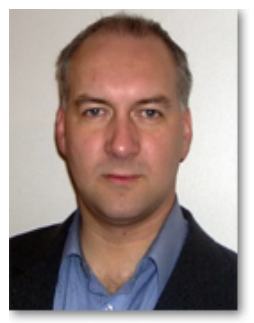

Miroslav Voznak is a professor with Department of Telecommunications, VSB-Technical University of Ostrava, Czech Republic. He received the Ph.D. degree in telecommunications technologies in 2002 from Faculty of Electrical Engineering and Computer Science, VSB - Technical University of Ostrava, his habilitation was finished in 2009 and he was appointed as a professor in 2017. He is IEEE Senior member, author or co-author more than hunderd articles in journals indexed in WoS. He serves as a member of editorial boards in several journals such as Sensors, Journal of Communications, and Advances in Electrical and Electronic Engineering. His major research interests are IP telephony, Speech quality, Wireless networks, Network security and Bigdata analytics. 